\title{
AUSTENITE AS A LOCAL MINIMIZER IN A MODEL OF MATERIAL MICROSTRUCTURE WITH A SURFACE ENERGY TERM*
}

\author{
J. J. BEVAN ${ }^{\dagger}$
}

\begin{abstract}
A family of integral functionals $\mathcal{F}$ which model in a simplified way material microstructure occupying a two-dimensional domain $\Omega$ and which take account of surface energy and a variable well depth is studied. It is shown that there is a critical well depth, whose scaling with the surface energy density and domain dimensions is given, below which the state $u=0$ is the global minimizer of a typical $F$ in $\mathcal{F}$. It is also shown that $u=0$ is a strict local minimizer of $F$ in the sense that if $v \neq 0$ is admissible and either $\|v\|_{L^{2}(\Omega)}$ or $\mathcal{L}^{2}\left(\left\{(x, y) \in \Omega:\left|v_{y}\right|(x, y) \geq 1\right\}\right)$ is sufficiently small (with quantitative bounds given in terms of the parameters appearing in the energy functional $F)$, then $F(v)>F(0)$. Provided the well depth is sufficiently large, the existence of a so-called energy barrier between $u=0$ and the global minimizer of $F$ is established under the assumption that paths $(v(t))_{0 \leq t \leq 1}$ connecting these two states obey $\left|v_{y}\right| \leq 1$ almost everywhere in the domain.
\end{abstract}

Key words. local minimizer, surface energy, energy barrier

AMS subject classifications. 49J40, 49J45, 74N15

DOI. $10.1137 / 080732699$

1. Introduction. The energy functionals we shall consider in this paper are related to the one used in [14] but with some important differences. The original Kohn-Müller functional is

$$
E_{K M}(u)=\int_{\Omega} \epsilon^{2} u_{y y}^{2}+\left(u_{y}^{2}-1\right)^{2}+u_{x}^{2} \mathrm{dx},
$$

where $\Omega=[0, L] \times[0,1], L>0, \epsilon>0$ is a small parameter which is sometimes referred to as the surface energy density, and $\mathrm{dx}$ is shorthand for $d \mathcal{L}^{2}(\mathrm{x})$. The addition of higher gradient terms, such as $\int_{\Omega} v_{y y}^{2} \mathrm{dx}$, has become a standard way of regularizing functionals of the form $\int_{\Omega} W(\nabla u) \mathrm{dx}$, where $W$ has multiple minima. See [3], [11], [8], [9], and [2] for further examples. More sophisticated regularizations, such as those detailed in [15] and [7], are available but are not considered in this paper.

Provided suitable boundary conditions are imposed, the global minimizers of $E_{K M}$ model in a simplified way the fine-scale microstructures that are observed to some degree at austenite-martensite interfaces in shape memory alloys. The second derivative term tempers the oscillations in the $y$-direction that any globally minimizing sequence will develop. Using a subtle argument, Kohn and Müller showed that the global minimization can be viewed as a straightforward competition between the term in $\int_{\Omega} u_{x}^{2} \mathrm{dx}$, effectively a measure of "spread," and a version of surface energy derived from the terms $\int_{\Omega} u_{y y}^{2} \mathrm{dx}$ and $\int_{\Omega}\left(u_{y}^{2}-1\right)^{2} \mathrm{dx}$, among functions $u$ with $\left|u_{y}\right|=1$ almost everywhere (a.e.). The result is that the infimum of the energy scales in $\epsilon$ as though it were evaluated at the now well-known branched microstructure. See [14] for details.

*Received by the editors August 12, 2008; accepted for publication (in revised form) February 14, 2011; published electronically April 27, 2011. This work was supported by the MULTIMAT program at the Max Planck Institute for Mathematics in the Natural Sciences, Leipzig, and by an RCUK Academic Fellowship at the University of Surrey.

http://www.siam.org/journals/sima/43-2/73269.html

${ }^{\dagger}$ Department of Mathematics, University of Surrey, Guildford, GU2 7XH, UK (j.bevan@surrey.ac. uk). 
However, $u=0$ is not a local minimizer of the functional $E_{K M}$ on sufficiently large domains $\Omega$, making $E_{K M}$ unsuitable for the study of austenite as a so-called metastable state, or for understanding the role played by well depth. This can be seen by an explicit calculation using the test function $v$ constructed in Proposition 4.3 below. The idea is to exploit the local concavity of the Kohn-Müller potential $W_{K M}(s, t)=s^{2}+\left(t^{2}-1\right)^{2}$ with respect to the $t$ variable at the point $(s, 0)$ for each fixed $s$, as follows.

Indeed, with the parameters $a$ and $d$ proportional to the size of the support of $v$ in the $x$ - and $y$-directions, respectively, it can be shown that

$$
E_{K M}(v)-E_{K M}(0)=\epsilon^{2} C_{1} \frac{d}{a}+C_{2} \frac{a^{3}}{d}-\frac{4}{9} a d
$$

for certain dimensionless constants $C_{1}$ and $C_{2}$. Choosing $a=\left(\frac{9 C_{1}}{2}\right)^{\frac{1}{2}} \epsilon$ and $d=$ $\left(\frac{81 C_{1} C_{2}}{2}\right)^{\frac{1}{2}} \epsilon$ then gives

$$
E_{K M}(v)-E_{K M}(0)=-\frac{3}{2} \epsilon^{2} C_{1} C_{2}^{\frac{1}{2}}
$$

Note that this choice of $a$ and $d$ is permissible provided $L \gg \epsilon$ and $1 \gg \epsilon$; we henceforth assume that $\Omega$ satisfies these conditions. Finally, one observes that $E_{K M}(\tau v)-$ $E_{K M}(0) \leq \tau^{2}\left(E_{K M}(v)-E_{K M}(0)\right)$ for real $\tau$ satisfying $|\tau| \leq 1$, leading to the conclusion that the functions $\tau v(x, y)$ are arbitrarily close to $u=0$ as $\tau \rightarrow 0$ and have lower energy as measured by $E_{K M}$. See section 4.2 for details of the function $v$. Consequently, we introduce below the functionals $E_{1}(\cdot ; \epsilon, \delta), E_{2}(\cdot ; \epsilon, \delta)$, and $E_{3}(\cdot ; \epsilon, \delta)$, each with a potential $W(s, t)$ that is in particular convex in a neighborhood of $(0,0)$. Besides this feature, $W$ enhances $W_{K M}$ by introducing a well depth; see below for details. The family of functionals $\mathcal{F}$ mentioned above consists of $E_{1}(\cdot ; \epsilon, \delta), E_{2}(\cdot ; \epsilon, \delta)$, and $E_{3}(\cdot ; \epsilon, \delta)$ defined below in (1.1), (1.2), and (1.3), respectively, as the parameters $\epsilon$ and $\delta$ vary.

DeFINITION 1.1.

$$
\begin{aligned}
E_{1}(u ; \epsilon, \delta) & =\int_{\Omega} \epsilon^{2} u_{y y}^{2}+u_{x}^{2} \mathrm{dx}+\delta \mathcal{L}^{2}(A(u)), \\
E_{2}(u ; \epsilon, \delta) & =\int_{\Omega} \epsilon^{2}\left|\nabla\left(u_{y}\right)\right|^{2}+u_{x}^{2} \mathrm{dx}+\delta \mathcal{L}^{2}(A(u)), \\
E_{3}(u ; \epsilon, \delta) & =\int_{\Omega} \epsilon^{2}|\nabla(\nabla(u))|^{2}+u_{x}^{2} \mathrm{dx}+\delta \mathcal{L}^{2}(A(u)), \\
B(u) & =\left\{(x, y) \in \Omega:\left|u_{y}(x, y)\right| \geq 1\right\}, \\
A(u) & =\Omega \backslash B(u) .
\end{aligned}
$$

In each case the functional takes the form

$$
E_{i}(u)=S_{i}(u)+\int_{\Omega} W_{\delta}(\nabla u) \mathrm{dx},
$$

where

$$
W_{\delta}(s, t)=s^{2}+\delta \chi_{(-1,1)}(t)
$$

and where $S_{i}(u)$ is a surface energy term. In particular, $S_{3}(u)$ will henceforth be written in the more conventional way

$$
S_{3}(u)=\int_{\Omega}\left|D^{2} u\right|^{2} \mathrm{dx}
$$


In the following we suppress the dependence of the $E_{i}$ on $\epsilon$ and $\delta$ for brevity. We study the behavior of each $E_{i}$ in the class $\mathcal{A}_{i}$ of admissible maps defined below. First we define the subclass of $W_{P e r}^{1,2}(\Omega, \mathbb{R}) \subset W^{1,2}(\Omega, \mathbb{R})$ of functions satisfying periodic boundary conditions (in the sense of trace) at the top and bottom of the domain $\Omega$ by

$$
W_{P e r}^{1,2}(\Omega, \mathbb{R})=\left\{u \in W^{1,2}(\Omega, \mathbb{R}): u(x, 1)=u(x, 0), \quad 0 \leq x \leq 1\right\} .
$$

Then

$$
\begin{aligned}
& \mathcal{A}_{1}=\left\{u \in W_{\text {Per }}^{1,2}(\Omega, \mathbb{R}): u_{y y} \in L^{2}(\Omega ; \mathbb{R}), u(0, y)=0,0 \leq y \leq 1\right\}, \\
& \mathcal{A}_{2}=\left\{u \in W_{\text {Per }}^{1,2}(\Omega, \mathbb{R}): u_{y} \in W^{1,2}(\Omega ; \mathbb{R}), u(0, y)=0,0 \leq y \leq 1\right\}, \\
& \mathcal{A}_{3}=\left\{u \in W_{\text {Per }}^{1,2}(\Omega, \mathbb{R}): \nabla u \in W^{1,2}(\Omega ; \mathbb{R}), u(0, y)=0,0 \leq y \leq 1\right\} .
\end{aligned}
$$

The new features of these models relative to the original Kohn-Müller functional are summarized here and discussed below:

(i) a variable well depth $\delta$ (see below for its definition);

(ii) a convex potential in a neighborhood of $(0,0)$;

(iii) the possibility of a cost, which may be zero, associated with the appearance of sets $B(u)$ of positive measure in $\Omega$, analogous to a lower bound on the cost of "nucleation" of martensite in austenite.

The term $\mathcal{L}^{2}(A(u))$ mimics the behavior of the term $\int_{\Omega}\left(u_{y}^{2}-1\right)^{2} \mathrm{dx}$ appearing in the Kohn-Müller functional $E_{K M}$ in the following sense. In order for $E_{i}(u)$ to approach its infimum it is necessary that $\left|u_{y}\right|<1$ occur only on a set of small measure: "most" values of $\left|u_{y}\right|$ will be close to or larger than 1. Looking at $E_{K M}$ and referring to their argument, we see that most values of the gradient $u_{y}$ of the global minimizer in that case will be near \pm 1 . The price we pay for replacing $\int_{\Omega}\left(u_{y}^{2}-1\right)^{2} \mathrm{dx}$ with a term proportional to $\mathcal{L}^{2}(A(u))$ is that large values of $\left|u_{y}\right|$ are not penalized as they would be in $E_{K M}$. In fact, the nature of the global minimizer of $E_{1}$ in $\mathcal{A}_{1}$ in a scaling sense can be deduced from the Kohn-Müller argument when $\delta$ is large enough, although we do not pursue this in the present work. When $\delta$ is in the range $\left(0, C \epsilon L^{-1}\right)$ for an appropriate dimensionless constant $C$ it happens that $u=0$ is the global minimizer of $E_{i}$ in $\mathcal{A}_{i}$ for $i=1,2,3$. We remark that this behavior with respect to varying the well depth $\delta$ may be an artifact of the choice of the periodic boundary conditions. In any case, it will be necessary to know just how large $\delta$ needs to be before $u=0$ ceases to be the global minimizer of $E_{i}$ in $\mathcal{A}_{i}$ for $i=1,2,3$.

The idea of introducing a well depth

$$
\delta=W_{\delta}(0,0)-W_{\delta}(0, \pm 1)
$$

comes from the Ball-James theory of martensitic phase transformations. See [3], [4] for details. The theory asserts that the stored-energy potential should change in a certain way as the temperature changes; we synthesize this by varying $\delta$, with $\delta=0$ corresponding to a high temperature stored-energy function and $\delta=1$, say, to a low temperature stored-energy function. The global minimizer in the case $\delta=0$ is $u=0$ which, in the full three-dimensional models, would be referred to as austenite. See [3], [4] for further details. We adopt the Ball-James approach by studying the static problem for each fixed well depth $\delta$; in particular, we do not attempt to introduce dynamics. It is shown in section 3 that $u=0$ is always a local minimizer of $E_{i}$ regardless of the size of $\delta$; the only effect $\delta$ has on local minimality is through the size 
of the neighborhood $\mathcal{N}_{i}$, say, of $u=0$ in $\mathcal{A}_{i}$ on which $u=0$ satisfies $E_{i}(v) \geq E_{i}(0)$ for all $v$ in $\mathcal{N}_{i}$ for $i=1,2,3$. See section 3, and in particular Theorem 3.1, for details. When the well depth is large enough we conjecture that it also appears in the scaling of the energy associated with the global minimizer; we do not pursue this in the present work. It is also natural to conjecture, and we thank an unnamed referee for doing so, that a suitably rescaled version of $E_{1} \Gamma$-converges to the Kohn-Müller functional

$$
I(u)=\int_{\Omega} \epsilon\left|u_{y y}\right|+u_{x}^{2} \mathrm{dx} .
$$

Here, $u_{y y}$ is a Radon measure of finite mass, and all admissible functions $u$ satisfy $\left|u_{y}\right|=1$ a.e. See [14, section 2] for details. Following the example of [8], it could be that for an appropriate choice of the constant $c$ the functionals

$$
\tilde{E}_{1}(u):=\frac{1}{\delta^{\frac{1}{2}}} \int_{\Omega} \epsilon^{2} u_{y y}^{2} \mathrm{dx}+c \delta^{\frac{1}{2}} \mathcal{L}^{2}(A(u))+\int_{\Omega} u_{x}^{2} \mathrm{dx}
$$

satisfy $\Gamma-\lim _{\delta \rightarrow \infty} \tilde{E}_{1}=I(u)$, defined in this case on those $u \in H^{1}(\Omega)$ for which $\left|u_{y}\right| \geq 1$ a.e. and $u_{y y}$ is a radon measure as described above. We have so far been unable to prove this and similar conjectures.

Note that the potential

$$
W_{\delta}(a, b)=a^{2}+\delta \chi_{(-1,1)}(b)
$$

is convex in a neighborhood of $(a, b)=(0,0)$. This is sufficient to establish that $u=0$ is an $L^{2}$-local minimizer of $E_{i}$ in $\mathcal{A}_{i}$. It is not necessary, though, as examples of Taheri show [16, section 4]. In fact, the potential $W_{\delta}$ can be bounded from below by a strongly convex potential in a neighborhood of zero by "borrowing" some surface energy and applying a suitable Poincaré inequality. For example, for any $\lambda \in(0,1)$, and in the case of $E_{2}$,

$$
E_{2}(u) \geq \int_{\Omega}(1-\lambda) \epsilon^{2}\left|\nabla u_{y}\right|^{2}+C_{\Omega} \lambda \epsilon^{2} u_{y}^{2}+W_{\delta}(\nabla u) \mathrm{dx}
$$

so that $\bar{W}_{\delta}(s, t):=W_{\delta}(s, t)+C_{\Omega} \lambda \epsilon^{2} t^{2}$ is strongly convex in a neighborhood of $(0,0)$. If we are allowed to vary the domain height, for example, by taking

$$
\Omega_{h}=[0, L] \times[0, h],
$$

then one can use the same procedure to bound the Kohn-Müller potential below by a potential that is strongly convex at $(0,0)$. The reason this works is that the Poincaré constant $C_{\Omega_{h}}$, say, becomes large when $h$ is small. See Taheri [16] for other interesting examples. When $h=1$ the Poincaré constant is not large enough for this trick to work, from which it follows easily that $u=0$ is not a local minimizer of $E_{K M}$ in $\mathcal{A}$ (provided $\epsilon$ is sufficiently small). We note that the idea of combining the strong convexity of $W$ with higher order terms in order to guarantee local minimality has been studied in [16] and [2]. See also [1]. Although we do not use these arguments directly, they are, in view of the comments above, one of the main reasons that we can expect $u=0$ to be a local minimizer of $E_{i}$ in $\mathcal{A}_{i}$ for $i=1,2,3$.

The results of section 3 are based on an apparently new inequality which relates all three terms appearing in $E_{1}$ and which, together with standard estimates, yields explicit functions $r(\epsilon, \delta)$ and $s(\epsilon, \delta)$ (given in (3.1) and (4.5), respectively) such that

$$
\|u\|_{L^{2}(\Omega)}<r(\epsilon, \delta) \text { or } \mathcal{L}^{2}(B(u))<s(\epsilon, \delta) \Longrightarrow E_{\Delta}(u)>E_{\Delta}(0),
$$


provided $u \neq 0$. It is doubtful whether the scalings involved are optimal, for reasons explained in section 3. Nevertheless, it is still a stronger and more explicit result than $L^{2}$-local minimality. The results for the functional $E_{1}$ are easily carried over to the functionals $E_{2}$ and $E_{3}$.

In section 4 we discuss the effect of modifying the surface energy term. The reasoning set out in section 4 points out that any path that connects $u=0$ with the global minimizer must, provided $\delta$ is large enough, pass through a state at which sets $B(u)$ of positive measure first appear. This is the basis for a calculation which tests whether such states automatically cost a certain minimum amount of energy to introduce, analogous to a "nucleation cost." Restricting our attention to those admissible $v$ that satisfy $\left|v_{y}\right| \leq 1$ a.e., Theorem 4.1 shows in particular that there is a constant $C$ depending only on the parameters $\epsilon$ and $L$ such that for each $M>0$ and each fixed $\sigma$ satisfying $\frac{1}{2}<\sigma<1$,

$$
E_{1}(v)-E_{1}(0) \geq C M\left(\mathcal{L}^{1}(\Pi(v))\right)^{\sigma+1}
$$

for all sufficiently small $\mathcal{L}^{1}(B(v))$. Here, $\Pi(v)$ is the projection of the set $B(v)$ onto the $x$-axis. When $\delta$ is sufficiently large, it can be inferred from this result that there is a strictly positive "energy barrier" separating the state $u=0$ from the global minimizer of $E_{1}$ in $\mathcal{A}_{1}$. Similar deductions can be made in the cases of $E_{2}$ and $E_{3}$. See section 4 for the details.

1.1. Notation. One- and two-dimensional Lebesgue measures are denoted, respectively, by $\mathcal{L}^{1}$ and $\mathcal{L}^{2}$ throughout the paper. The usual Sobolev space notation $W^{k, p}$ is used to denote the class of $k$-times weakly differentiable $L^{p}$ functions all of whose derivatives lie in $L^{p}(\Omega)$. The $L^{p}$ norm of a function $v$ is denoted by $\|v\|_{p}$ or $\|v\|_{L^{p}(\Omega)}$, depending on the context. The class of absolutely continuous functions is denoted $A C$, and those functions which are absolutely continuous along almost all lines parallel to the coordinate axes are written $A C L$. See [17] for further details. The characteristic function of any set $S$ is written $\chi_{S}$. All other notation is standard, with the possible exception that the value of the dimensionless positive constants $C$ appearing in various inequalities may, where no confusion arises, change from line to line. Where it is necessary to distinguish between positive constants we shall use the convention that if $C$ and $c$ appear in the same calculation, then $c<C$. We will also employ the convention that roman letters x represent vectors in $\mathbb{R}^{2}$.

2. The effect of a variable well depth. When $\delta=0$ it is clear by inspection that $u=0$ is a global minimizer of $E_{i}$ in $\mathcal{A}_{i}$ for $i=1,2,3$. Therefore the following definition makes sense.

Definition 2.1. For each $i=1,2,3$,

$$
\delta_{i}=\sup \left\{\delta \geq 0: u=0 \text { globally minimizes } E_{i} \text { in } \mathcal{A}_{i}\right\} .
$$

Each $\delta_{i}$ will be referred to as a critical well depth. The ordering

$$
E_{1}(v) \leq E_{2}(v) \leq E_{3}(v)
$$

for all appropriate $v$, together with the fact that $E_{1}(0)=E_{2}(0)=E_{3}(0)$ for fixed $\epsilon$ and $\delta$, implies the inclusions

$$
\begin{aligned}
& \left\{\delta: E_{1}(v) \geq E_{1}(0) \forall v \in \mathcal{A}_{1}\right\} \subset\left\{\delta: E_{2}(v) \geq E_{2}(0) \forall v \in \mathcal{A}_{2}\right\}, \\
& \left\{\delta: E_{2}(v) \geq E_{2}(0) \forall v \in \mathcal{A}_{2}\right\} \subset\left\{\delta: E_{3}(v) \geq E_{3}(0) \forall v \in \mathcal{A}_{3}\right\},
\end{aligned}
$$


and hence

$$
\delta_{1} \leq \delta_{2} \leq \delta_{3}
$$

It will be shown in this section that all the $\delta_{i}$ scale alike with respect to $\epsilon$ and $L$ in the sense that there are dimensionless constants $c<C$ such that

$$
\frac{c \epsilon}{L} \leq \delta_{1} \leq \delta_{2} \leq \delta_{3} \leq \frac{C \epsilon}{L},
$$

provided $\epsilon$ is sufficiently small.

To begin with, Proposition 2.5 below shows that there is a constant $C$ depending only on $L$ such that $\delta_{1} \geq C \epsilon^{2}$. This follows relatively simply by using only the surface energy term in the functional $E_{1}$, and it turns out to be a crude lower bound on $\delta_{1}$. A better (i.e., larger) lower bound is obtained in section 2.2, where it is shown that

$$
\delta_{1} \geq \frac{c \epsilon}{L} .
$$

The optimality (in terms of its scaling in $\epsilon$ and $L$ ) of this lower bound is proved by evaluating the energy of a particular element $v^{\epsilon}$ of $\mathcal{A}_{3}$ in section 2.3. The structure of $v^{\epsilon}$ is given in section 2.3. It is not a branching microstructure, which is the global minimizer of the model studied by Kohn and Müller (corresponding to the case $\delta_{i} \sim 1$ in the models studied in this paper).

The main result of this part of the paper is as follows.

TheOREM 2.2. With $E_{i}, \mathcal{A}_{i}$, and $\delta_{i}$ as above, there are dimensionless constants $c<C$ and $\epsilon_{0}>0$ such that

$$
\frac{c \epsilon}{L} \leq \delta_{i} \leq \frac{C \epsilon}{L} \text { if } 0<\epsilon<\epsilon_{0} .
$$

Moreover, for all $\delta$ the global minimizer $U_{i}$ of $E_{i}$ in $\mathcal{A}_{i}$ exists, and when $\frac{\delta}{\delta_{i}}$ is large enough it satisfies $\mathcal{L}^{2}\left(B\left(U_{i}\right)\right)>0$.

The proof of Theorem 2.2 rests on Propositions 2.6 and 2.8 in sections 2.2 and 2.3 below. The lower bound is proved with the help of an interpolation inequality; the upper bound corresponds to the statement concerning the optimality (in a scaling sense) of the lower bound. The proof of the existence of the global minimizer $U_{i}$ of $E_{i}$ in $\mathcal{A}_{i}$ is a relatively straightforward application of the direct method of the calculus of variations. It is given in an appendix for the sake of completeness. The last assertion of Theorem 2.2 can be deduced from the comparison function constructed during the proof of Proposition 2.8.

2.1. A simple lower bound on $\delta_{1}$. We recall that

$$
E_{1}(u)=\int_{\Omega} \epsilon^{2} u_{y y}^{2}+u_{x}^{2} \mathrm{dx}+\delta \mathcal{L}^{2}(A(u))
$$

where

$$
A(u)=\left\{(x, y) \in \Omega:\left|u_{y}(x, y)\right|<1\right\} .
$$

The following lemma will be used to show that $\delta_{1}$ is strictly positive; it will also play an important role in section 3. 
Lemma 2.3. Let $u \in \mathcal{A}_{1}$ satisfy $\mathcal{L}^{2}(B(u))>0$ and define for each $x \in[0, L]$

$$
\begin{aligned}
l_{x} & =\{x\} \times[0,1], \\
\Pi(u) & =\left\{x \in[0, L]: \mathcal{L}^{1}\left(l_{x} \cap B(u)\right)>0\right\} .
\end{aligned}
$$

Then

$$
\frac{\int_{\Omega} u_{y y}^{2} \mathrm{dx}}{\mathcal{L}^{2}(B(u))} \geq \frac{4}{\tau(1-\tau)}
$$

where

$$
\tau:=\frac{\mathcal{L}^{2}(B(u))}{\mathcal{L}^{1}(\Pi(B(u)))} .
$$

Proof. We begin by remarking that the quantity on the right-hand side of (2.5) is finite under the assumptions of the lemma. Let $B=B(u)$ for brevity. Clearly, $\mathcal{L}^{2}(B) \neq 0$ implies $\tau>0$. By Fubini's theorem we have that $\tau \leq 1$. Furthermore, the membership of $u$ in $\mathcal{A}_{1}$ implies $\tau<1$. If not, then for almost all $x \in[0, L]$ the lines $l_{x}$ would satisfy

$$
\mathcal{L}^{1}\left(l_{x} \cap B\right)=1 .
$$

Then, since $u_{y}$ is absolutely continuous along almost all lines $l_{x}$, we must have for almost all $x$ that either

$$
u_{y}(x, y) \geq 1 \text { for a.e. } y \in[0,1]
$$

or

$$
u_{y}(x, y) \leq-1 \text { for a.e. } y \in[0,1],
$$

contradicting the periodic boundary conditions imposed along $y=0$ and $y=1$. In fact, this argument shows that $\mathcal{L}^{1}\left(l_{x} \cap B\right)<1$ for almost all $x$.

Now we proceed with the proof of inequality (2.5). Let $x \in \Pi(u)$. The argument above shows that we may assume there is at least one open set $\mathcal{Y}_{1} \subset l_{x} \cap B$ on which $u_{y}(x, \cdot) \geq 1$ and at least one other open set $\mathcal{Y}_{2} \subset l_{x} \cap B$ on which $u_{y} \leq-1$. We may suppose that $y_{1}:=\sup \mathcal{Y}_{1}<\inf \mathcal{Y}_{2}=: y_{2}$, so that the intervening set is $\left[y_{1}, y_{2}\right]$. It is easy to check that the minimum of the scalar functional

$$
f \mapsto \int_{y_{1}}^{y_{2}}\left(f^{\prime \prime}\right)^{2} d y
$$

among $f \in W^{2,2}\left(\left[y_{1}, y_{2}\right], \mathbb{R}\right)$ satisfying $f^{\prime}\left(y_{1}\right) \geq 1$ and $f^{\prime}\left(y_{2}\right) \leq-1$ is $\frac{4}{y_{2}-y_{1}}$. The minimization calculation is of the "free endpoint" kind, so that it prescribes optimal values for the differences $f\left(y^{*}\right)-f\left(y_{1}\right)$ and $f\left(y_{2}\right)-f\left(y^{*}\right)$, where $y_{1}<y^{*}<y_{2}$ satisfies $f^{\prime}\left(y^{*}\right)=0$. Note that $y^{*}$ exists because $v_{y} \in \mathrm{AC}\left(l_{x}\right)$ by assumption. The minimizer is $f(y)=\frac{\left(y-\frac{\left(y_{1}+y_{2}\right)}{2}\right)^{2}}{y_{1}-y_{2}}$, with $y^{*}=\frac{y_{1}+y_{2}}{2}$. Since this calculation is elementary we omit the proof.

The result is

$$
\int_{l_{x}} u_{y y}{ }^{2}(x, y) d y \geq \frac{4}{y_{2}-y_{1}}
$$


But since

$$
\begin{aligned}
y_{2}-y_{1} & \leq \mathcal{L}^{1}\left(A \cap l_{x}\right), \\
\mathcal{L}^{1}\left(A \cap l_{x}\right) & =1-\mathcal{L}^{1}\left(B \cap l_{x}\right)
\end{aligned}
$$

we must have

$$
\int_{l_{x}} u_{y y}{ }^{2}(x, y) d y \geq \frac{4}{1-\mathcal{L}^{1}\left(B \cap l_{x}\right)} .
$$

Integrating over $x \in \Pi(u)$ and applying Jensen's inequality gives

$$
\int_{\Omega} u_{y y}^{2} \mathrm{dx} \geq \frac{4 \mathcal{L}^{1}(\Pi(u))^{2}}{\int_{\Pi(u)} 1-\mathcal{L}^{1}\left(B \cap l_{x}\right) d x} .
$$

Dividing by $\mathcal{L}^{2}(B)$ and rearranging yields inequality (2.5).

Remark 1. The global minimizer $v$ of the functional on the left-hand side of (2.5) is such that $\tau=\frac{1}{2}$. Though easy to construct, $v$ can never belong to $\mathcal{A}_{1}$ because it violates the boundary condition $u=0$ at $x=0$.

In the rest of the paper it will be useful to have a label for those elements $u$ of $\mathcal{A}_{i}$ for which $\mathcal{L}^{2}(B(u))>0$ holds.

Definition 2.4. $\mathcal{A}_{i}^{+}:=\left\{u \in \mathcal{A}_{i}: \mathcal{L}^{2}(B(u))>0\right\}$.

Proposition 2.5. $\delta_{1} \geq C_{2} \epsilon^{2} \geq C_{1} \epsilon^{2}>0$, where

$$
\begin{aligned}
& C_{1}=\inf \left\{\frac{\int_{\Omega} u_{y y}^{2} \mathrm{dx}}{\int_{\Omega} u_{y}^{2} \mathrm{dx}}: u \in \mathcal{A}_{1}^{+}\right\}, \\
& C_{2}=\inf \left\{\frac{\int_{\Omega} u_{y y}^{2} \mathrm{dx}}{\mathcal{L}^{2}(B(u))}: u \in \mathcal{A}_{1}^{+}\right\} .
\end{aligned}
$$

Proof. By Chebyshev's inequality, $C_{2} \geq C_{1}$. Therefore we need only prove $\delta_{1} \geq$ $C_{2} \epsilon^{2}$. By Remark 1 above, $C_{2}=16$, but the infimum is not attained. Now

$$
\begin{aligned}
E_{1}(u)-E_{1}(0) & =\int_{\Omega} \epsilon^{2} u_{y y}^{2}+u_{x}^{2} \mathrm{dx}-\delta \mathcal{L}^{2}(B) \\
& \geq\left(\frac{\int_{\Omega} \epsilon^{2} u_{y y}^{2} \mathrm{dx}}{\mathcal{L}^{2}(B)}-\delta\right) \mathcal{L}^{2}(B) \\
& \geq\left(16 \epsilon^{2}-\delta\right) \mathcal{L}^{2}(B) .
\end{aligned}
$$

So if $\delta \leq 16 \epsilon^{2}$, then $u=0$ is a global minimizer of $E_{1}$, and hence $\delta_{1} \geq 16 \epsilon^{2}$.

2.2. A refined lower bound on $\delta_{1}$. In this section we show that there is a dimensionless constant $c$ such that $\delta_{1} \geq \frac{c \epsilon}{L}$ for all $\epsilon$. This improves (i.e., increases) the lower bound obtained in section 2.1. The reason for the improvement is essentially that the term $\int_{\Omega} u_{x}^{2} \mathrm{dx}$ is brought into play.

We shall make use of the standard interpolation inequality

$$
\frac{1}{\sigma^{2}} \int_{0}^{1} f_{y y}^{2} d y+\sigma^{2} \int_{0}^{1}(f-\rho)^{2} d y \geq C \int_{0}^{1} f_{y}^{2} d y
$$


which holds for some $C>0$, all $\rho$, all nonzero $\sigma$, and all $f \in W^{2,2}([0,1], \mathbb{R})$. (See, e.g., [12, section 7.12].) Let $u \in \mathcal{A}_{1}$, fix $x \in \Pi(u)$, and take $\rho=0, f(y)=u(x, y)$ in (2.8) above. Using the inequality

$$
\int_{0}^{1} u_{y}^{2}(x, y) d y \geq \mathcal{L}^{1}\left(l_{x} \cap B(u)\right)
$$

integrating over $x \in \Pi(u)$, and using Fubini's theorem, we obtain

$$
\frac{1}{\sigma^{2}} \int_{\Omega} u_{y y}^{2} \mathrm{dx}+\sigma^{2} \int_{\Omega} u^{2} \mathrm{dx} \geq C \mathcal{L}^{2}(B(u)) .
$$

Minimizing the left-hand side of (2.9) over nonzero $\sigma$, we see that

$$
\left(\int_{\Omega} u_{y y}^{2} \mathrm{dx}\right)^{\frac{1}{2}}\left(\int_{\Omega} u^{2} \mathrm{dx}\right)^{\frac{1}{2}} \geq \frac{C \mathcal{L}^{2}(B(u))}{2} .
$$

Note that the constant $C$ is independent of the dimensions of the domain $\Omega$.

We also need the standard Poincaré inequality

$$
\int_{\Omega} u_{x}^{2} \mathrm{dx} \geq \frac{C}{L^{2}} \int_{\Omega} u^{2} \mathrm{dx}
$$

which uses the boundary condition $u=0$ along $x=0$. The constant $C$ is independent of the domain dimensions.

Proposition 2.6. There is a dimensionless constant $c>0$ such that $\delta_{1} \geq \frac{c \epsilon}{L}$ for all $\epsilon>0$. In particular, the lower bound on $\delta_{1}$ stated in Theorem 2.2 holds.

Proof. Let $u \in \mathcal{A}_{1}$ and set $B=B(u)$. By definition of $E_{1}$, and from inequalities (2.10) and (2.11), we have

$$
\begin{aligned}
E_{1}(u)-E_{1}(0) & =\left(\frac{\int_{\Omega} \epsilon^{2} u_{y y}^{2} \mathrm{dx}+\int_{\Omega} u_{x}^{2} \mathrm{dx}}{\mathcal{L}^{2}(B)}-\delta\right) \mathcal{L}^{2}(B) \\
& \geq\left(\frac{C \epsilon^{2} \mathcal{L}^{2}(B)}{4 \int_{\Omega} u^{2} \mathrm{dx}}+\frac{C \int_{\Omega} u^{2} \mathrm{dx}}{L^{2} \mathcal{L}^{2}(B)}-\delta\right) \mathcal{L}^{2}(B)
\end{aligned}
$$

Letting

$$
t=\frac{\int_{\Omega} u^{2} \mathrm{dx}}{\mathcal{L}^{2}(B)}
$$

we see that the right-hand side of (2.12) above has the form

$$
\left(\frac{C \epsilon^{2}}{4 t}+\frac{C t}{L^{2}}-\delta\right) \mathcal{L}^{2}(B)
$$

The term in parentheses is minimized when $t=c \epsilon L$ for some constant $c$. From this it follows that any $\delta \leq \frac{c \epsilon}{L}$ is such that $u=0$ is a global minimizer of $E_{1}$. Therefore $\delta_{1} \geq \frac{c \epsilon}{L}$.

In some cases one can do better than Proposition 2.6. The following lemma shows that the lower bound on $\delta_{1}$ obtained above is correct with constant $c=1$, provided condition (2.13) below holds. This supplementary condition amounts to a strengthening of the boundary condition along $y=0$ and $y=1$; it is satisfied, for 
example, by all sufficiently smooth admissible functions having compact support in $\Omega$.

Lemma 2.7. Let $v \in \mathcal{A}_{1}^{+} \cap\left\{u \in H^{1}(\Omega): u_{y} \in H^{1}(\Omega)\right\}$ satisfy

$$
\int_{l_{x}}\left(v_{y} v_{x}\right)_{y} d y=0
$$

for a.e. $x$ in $[0, L]$. Then for a.e. $x$ in $\Pi(v)$

$$
\int_{\Omega} \epsilon^{2} v_{y y}^{2}+v_{x}^{2} \mathrm{dx} \geq \epsilon \mathcal{L}^{1}\left(l_{x} \cap B\right) .
$$

From this it follows that $\delta_{1} \geq \frac{\epsilon}{L}$.

Furthermore, if $v \in \mathcal{A}_{2}$ satisfies

$$
\int_{\Omega} v_{y x}^{2} \mathrm{dx} \geq \frac{1}{\epsilon^{2}}\left(\delta-\frac{\epsilon}{\mathcal{L}^{1}(\Pi(v))}\right) \mathcal{L}^{2}(B),
$$

then

$$
E_{2}(v) \geq E_{2}(0)
$$

Proof. First fix $x \in \Pi(v)$ for which (2.13) holds. Then

$$
\begin{aligned}
\int_{\Omega} \epsilon^{2} v_{y y}^{2}+v_{x}^{2} \mathrm{dx} & \geq \int_{[0, x] \times[0,1]} \epsilon^{2} v_{y y}^{2}+v_{x}^{2} \mathrm{dx} \\
& \geq 2 \epsilon\left|\int_{\Omega} v_{y y} v_{x} \mathrm{dx}\right| \\
& =2 \epsilon\left|\int_{\Omega}\left(v_{y} v_{x}\right)_{y}-v_{y} v_{y x} \mathrm{dx}\right| \\
& =2 \epsilon\left|\int_{0}^{x}\left\{\int_{l_{x^{\prime}}}\left(v_{y} v_{x}\right)_{y} d y-\int_{l_{x^{\prime}}}\left(\frac{1}{2} v_{y}^{2}\right)_{x} d y\right\} d x^{\prime}\right| \\
& =\epsilon \int_{l_{x}} v_{y}^{2} d y \\
& \geq \epsilon \mathcal{L}^{1}\left(l_{x} \cap B\right)
\end{aligned}
$$

where we have applied (2.13) and the boundary condition $v(0, y)=0$ for $0 \leq y \leq 1$ to pass from the fourth to the fifth line. Integrating both sides of inequality (2.14) over $\Pi(v)$, dividing by $\mathcal{L}^{1}(\Pi(v))$, and inserting the resulting expression into the definition of $E_{1}(v)$ gives

$$
E_{1}(v)-E_{1}(0) \geq\left(\frac{\epsilon}{\mathcal{L}^{1}(\Pi(v))}-\delta\right) \mathcal{L}^{2}(B)
$$

from which the inequality $\delta_{1} \geq \frac{\epsilon}{L}$ follows easily.

Inserting the integrated from of (2.14) into $E_{2}(v)$ gives

$$
E_{2}(v)-E_{2}(0) \geq \epsilon^{2} \int_{\Omega} v_{y x}^{2} \mathrm{dx}+\left(\frac{\epsilon}{\mathcal{L}^{1}(\Pi(v))}-\delta\right) \mathcal{L}^{2}(B)
$$

Therefore (2.15) implies $E_{2}(v) \geq E_{2}(0)$, as required.

Remark 2. Any $\delta$ satisfying $\delta \leq \frac{\epsilon}{L}$ forces (2.15) to hold. Therefore inequality (2.15) provides a shortcut to the proof that $\delta_{2} \geq \frac{\epsilon}{L}$ whenever (2.13) is true. 
2.3. A sharp upper bound on $\boldsymbol{\delta}_{\mathbf{3}}$. We show in this section that there is a constant $C$ independent of $\Omega$ and $\epsilon$ such that $\delta_{3} \leq \frac{C \epsilon}{L}$ if $\epsilon$ is sufficiently small. The idea of the proof can be explained as follows. Let us suppose that for each $\epsilon>0$ there is an element $v^{\epsilon}$ of $\mathcal{A}_{3}$ with the properties that

$$
\begin{aligned}
\int_{\Omega} \epsilon^{2}\left|D^{2} v^{\epsilon}\right|^{2}+\left(v_{x}^{\epsilon}\right)^{2} \mathrm{dx} & \leq C_{1} \epsilon, \\
\mathcal{L}^{2}\left(B\left(v^{\epsilon}\right)\right) & \geq C_{2} L .
\end{aligned}
$$

The constants $C_{1}$ and $C_{2}$ should not depend on $\epsilon$ or $L$. Let $\delta<\delta_{3}$. Then in particular

$$
E_{3}\left(v^{\epsilon}\right)-E_{3}(0) \geq 0 .
$$

Using (2.16) and (2.17) above,

$$
C_{1} \epsilon-\delta C_{2} L \geq E_{3}\left(v^{\epsilon}\right)-E_{3}(0) .
$$

Thus $\delta \leq \frac{C_{1} \epsilon}{C_{2} L}$ whenever $\delta<\delta_{3}$. Letting $\delta \rightarrow \delta_{3}$ yields the desired upper bound. It remains to prove the existence of a map $v^{\epsilon} \in \mathcal{A}_{3}$ with the properties (2.16) and (2.17).

Proposition 2.8. There exists a map $v^{\epsilon}$ in the class $\mathcal{A}_{3}$ and dimensionless constants $C_{1}$ and $C_{2}$ such that (2.16) and (2.17) hold. In particular, there is a dimensionless constant $C$ such that

$$
\delta_{3} \leq \frac{C \epsilon}{L}
$$

whenever $\epsilon$ is sufficiently small, proving the upper bound on $\delta_{3}$ stated in Theorem 2.2 .

Proof. Let $k, l, h>0$ and define $H:[0, l] \rightarrow \mathbb{R}$ by

$$
H(x)=h-k x,
$$

where $k l=\frac{h}{2}$. Define the function $w$ on $[0, l] \times[0,2 h]$ by

$$
w(x, y)= \begin{cases}\frac{y^{2}}{2 H(x)} & \text { if } 0 \leq x \leq l, 0<y \leq H(x), \\ y-\frac{H(x)}{2} & \text { if } 0 \leq x \leq l, H(x) \leq y \leq 2 h-H(x), \\ 2 h-\frac{3 H(x)}{2}-\frac{(y-2 h)^{2}}{2 H(x)} & \text { if } 0 \leq x \leq l, 2 h-H(x) \leq y \leq 2 h .\end{cases}
$$

Now extend $w$ to $[0, l] \times[0,4 h]$ by reflection in the line $y=2 h$, namely,

$$
w(x, y)=w(x, 4 h-y) \quad \text { if } 0 \leq x \leq l, 2 h \leq y \leq 4 h .
$$

It can be checked that

$$
\begin{aligned}
\int_{0}^{4 h} \int_{0}^{l}\left|D^{2} w\right|^{2} \mathrm{dx} & =\frac{1}{k}\left(c_{1}+c_{2} k^{2}+c_{3} k^{4}\right), \\
\int_{0}^{4 h} \int_{0}^{l} w_{x}^{2} \mathrm{dx} & =c_{3} k^{2} l h
\end{aligned}
$$

where the $c_{i}$ are positive dimensionless constants whose precise values are not important. Suppose $h$ is chosen so that $N:=\frac{1}{4 h}$ is a positive integer. Extend $w$ by 
periodicity to $[0, l] \times[0,1]$ and label the resulting function $w$ again. A computation using (2.18) above together with $k l=\frac{h}{2}$ gives

$$
\begin{aligned}
\int_{0}^{1} \int_{0}^{l}\left|D^{2} w\right|^{2} \mathrm{dx} & =\frac{1}{h^{2}}\left(c_{1}+c_{2}\left(\frac{h}{l}\right)^{2}+c_{3}\left(\frac{h}{l}\right)^{4}\right), \\
\int_{0}^{1} \int_{0}^{l} w_{x}^{2} \mathrm{dx} & =c_{4} \frac{h^{2}}{l}
\end{aligned}
$$

Clearly $w$ is not an element of $\mathcal{A}$ because it doesn't satisfy the boundary condition at $x=0$. But we can interpolate between $w(0, y)$ and the function $y \mapsto 0$ as follows. Define $v:[0, l] \times[0,1] \rightarrow \mathbb{R}$ by $v(x, y)=\frac{x}{l} w(0, y)$ and compute directly.

$$
\begin{aligned}
\int_{0}^{1} \int_{0}^{l}\left|D^{2} v\right|^{2} \mathrm{dx} & =\frac{1}{h}\left(c_{4} \frac{l}{h}+c_{5} \frac{h}{l}\right), \\
\int_{0}^{1} \int_{0}^{l} v_{x}^{2} \mathrm{dx} & =c_{6} \frac{h^{2}}{l} .
\end{aligned}
$$

By construction $v$ and $w$ depend only on the parameters $h$ and $l$. The last step is to glue $v$ and $w$ together to give an element $v^{\epsilon}$ of $\mathcal{A}$. Define

$$
v^{\epsilon}(x, y)= \begin{cases}v(x, y ; h, l), & 0 \leq x \leq l, 0 \leq y \leq 1 \\ w(x-l, y ; h, l), & l \leq x \leq 2 l, 0 \leq y \leq 1 .\end{cases}
$$

See Figure 1 for a graphical indication of this function. It is straightforward to check that

$$
\mathcal{L}^{2}\left(B\left(v^{\epsilon}\right)\right)=\frac{l}{8}
$$

Finally we use (2.20)-(2.24) to compute

$$
\begin{aligned}
E_{3}\left(v^{\epsilon}\right)-E_{3}(0) & =\frac{\epsilon^{2}}{h^{2}}\left(c_{1}+c_{2}\left(\frac{h}{l}\right)^{2}+c_{3}\left(\frac{h}{l}\right)^{4}+c_{4} l+c_{5} \frac{h^{2}}{l}\right) \\
& +c_{6} \frac{h^{3}}{l}+c_{7} \frac{h^{2}}{l}-\frac{\delta l}{8} .
\end{aligned}
$$

The domain of $v^{\epsilon}$ is $\Omega$, provided we choose $l=\frac{L}{2}$. Choosing $\frac{h^{2}}{L}=c^{2} \epsilon$, where $c$ is such that $\left(4 c(\epsilon L)^{\frac{1}{2}}\right)^{-1} \in \mathbb{N}$ and $|c-1|$ is minimized, and inserting into the above gives

$$
\begin{aligned}
E_{3}\left(v^{\epsilon}\right)-E_{3}(0) & =\frac{\epsilon}{c^{2} L}\left(c_{1}+c_{2} \frac{\epsilon}{L}+c_{3}\left(\frac{\epsilon}{L}\right)^{2}+c_{4} L+c_{5} \epsilon\right) \\
& +c_{6} \epsilon^{\frac{3}{2}} L^{\frac{1}{2}}+c_{7} \epsilon-\frac{\delta L}{16} .
\end{aligned}
$$

By ignoring the term in $\delta$ it can immediately be seen that (2.16) is satisfied. From (2.24) we have $\mathcal{L}^{2}\left(B\left(v^{\epsilon}\right)\right)=\frac{L}{16}$, so $(2.17)$ holds. By the reasoning set out in the lines following (2.16) and (2.17), this concludes the proof.

Remark 3. The proof of the upper bound on $\delta_{3}$ can be obtained directly from $(2.25)$ as follows. Simply note that the inequality $E_{3}\left(v^{\epsilon}\right)-E_{3}(0) \geq 0$ holds because $\delta<\delta_{3}$ has been assumed. In view of (2.25), this gives $\delta \leq \frac{C \epsilon}{L}$. Hence $\delta_{3} \leq \frac{C \epsilon}{L}$. 


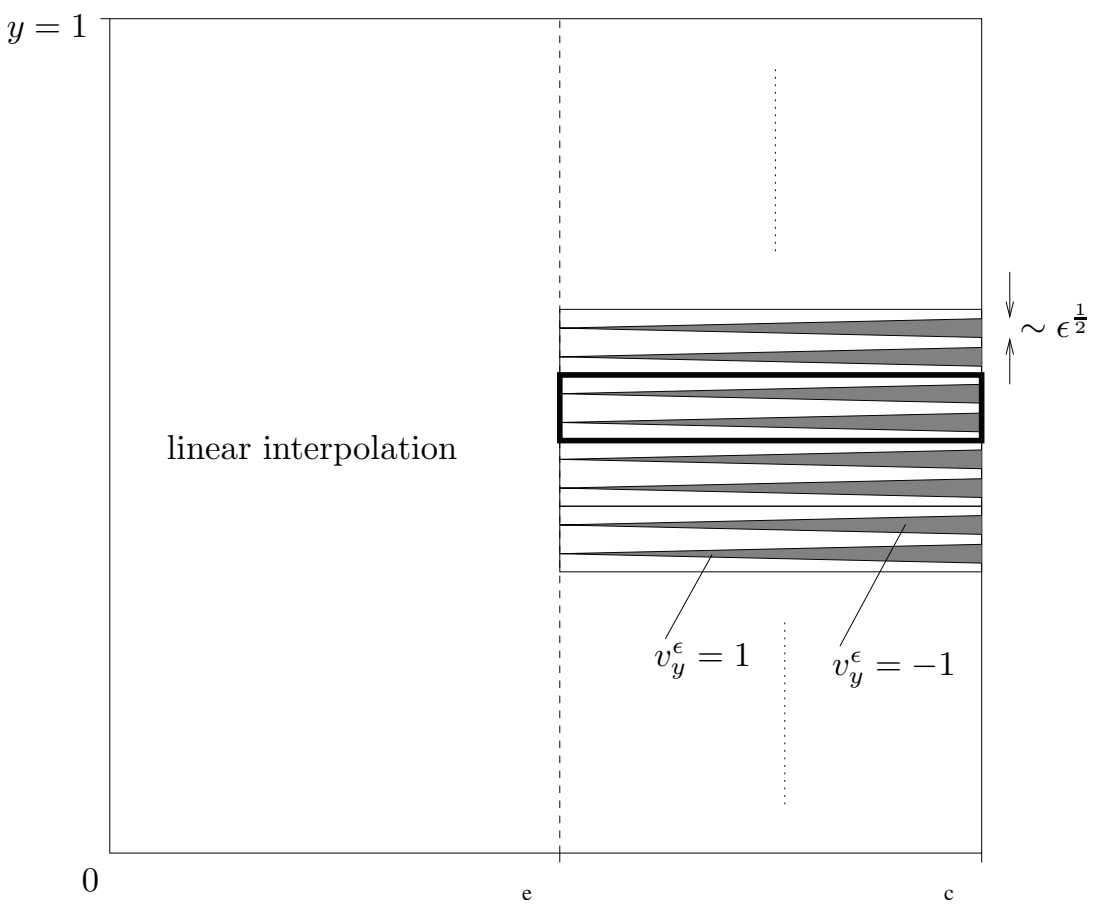

Fig. 1. The construction of the test function $v^{\epsilon} .\left|v_{y}^{\epsilon}\right|=1$ in the shaded regions; $\left|v_{y}^{\epsilon}\right|<1$ in all other areas. The basic repeating unit shown with a bold outline in the region $\frac{L}{2} \leq x \leq L$ has a vertical lengthscale of order $\epsilon^{\frac{1}{2}}$.

3. Austenite as a local minimizer of $\boldsymbol{E}_{\boldsymbol{i}}$. We saw earlier that $u=0$ is a global minimizer of $E_{i}$ in $\mathcal{A}_{i}$, provided $\delta \leq \delta_{i}$. In this section we show that when $\delta>\delta_{i}$ the state $u=0$ is a local minimizer of $E_{i}$ in $\mathcal{A}_{i}$ under the conditions set out in Theorem 3.1 below. It suffices to prove the theorem for the functional $E_{1}$ in view of the ordering (2.1) and since $E_{1}(0)=E_{2}(0)=E_{3}(0)$. The result is then automatically true for the other $E_{i}$.

THEOREM 3.1. Let $\epsilon>0$ and $\delta>0$. There exists a constant $C$ independent of $\epsilon$ and $\delta$ such that if the nonzero function $v \in \mathcal{A}_{1}$ satisfies either

$$
\|v\|_{L^{2}(\Omega)}<C \epsilon^{\frac{7}{2}} \delta^{-2}
$$

or

$$
\mathcal{L}^{2}(B(v))<C \epsilon^{6} \delta^{-4} L^{-1}
$$

then

$$
E_{1}(v)>E_{1}(0)
$$

The same statement holds with $E_{i}$ and $\mathcal{A}_{i}$ in place of $E_{1}$ and $\mathcal{A}_{1}$, respectively, for $i=2,3$.

The proof has elements in common with [2, Theorem 2.2]. A key difference, however, is that here we take into account the size of the set $B(u)$, where $\left|u_{y}\right| \geq 1$. According to the theorem, $u=0$ is a strict local minimizer in the sense that the strict 
inequality $E_{i}(u)>E_{i}(0)$ holds whenever $\|u\|_{L^{2}(\Omega)}$ or $\mathcal{L}^{2}(B(u))$ is sufficiently small and nonzero, with bounds given explicitly in terms of the parameters $\epsilon, \delta$, and $L$. See section 4 for a stronger version of Theorem 3.1. It would be interesting to determine whether, in the terminology of Ball and colleagues [5], [6], $u=0$ lies in an energy well of the $E_{i}$ with respect to either $\|v\|_{2}$ or $\mathcal{L}^{2}(B(v))$.

We note that for any $v \in \mathcal{A}$

$$
\left(\int_{l_{x}} v_{y y}^{2} d y\right)^{\frac{1}{2}}\left(\int_{l_{x}} v^{2} d y\right)^{\frac{1}{2}} \geq C \mathcal{L}^{1}\left(l_{x} \cap B\right)
$$

for a.e. $x \in[0, L]$. This follows by applying the simple lower bound $\int_{l_{x}} v_{y}^{2} d y \geq$ $\mathcal{L}^{1}\left(l_{x} \cap B\right)$ to the interpolation inequality $(2.8)$.

We wish to prove $E_{1}(v)-E_{1}(0)>0$, provided $v \neq 0$ and that either $\int_{\Omega} v^{2} \mathrm{dx}$ or $\mathcal{L}^{2}(B(u))$ is small enough. It is clear that if either

$$
\int_{\Omega} v_{y y}^{2} \mathrm{dx}>\frac{\delta}{\epsilon^{2}} \mathcal{L}^{2}(B)
$$

or

$$
\int_{\Omega} v_{x}^{2} \mathrm{dx}>\delta \mathcal{L}^{2}(B)
$$

then there is nothing to prove. There is also nothing to prove should $\mathcal{L}^{2}(B)=0$, since in this case the assumption $v \neq 0$ implies (in view of the boundary conditions) that $\int_{\Omega} \epsilon^{2} v_{y y}^{2}+v_{x}^{2} \mathrm{dx}>0$, and hence that $E_{1}(v)-E_{1}(0)>0$. Thus we may assume that

$$
\int_{\Omega} v_{y y}^{2} \mathrm{dx} \leq \frac{\delta}{\epsilon^{2}} \mathcal{L}^{2}(B)
$$

and

$$
\int_{\Omega} v_{x}^{2} \mathrm{dx} \leq \delta \mathcal{L}^{2}(B)
$$

where $\mathcal{L}^{2}(B)>0$.

The claim will be that the two conditions (3.6) and (3.7) imply lower bounds on $\int_{\Omega} v^{2} \mathrm{dx}$ and $\mathcal{L}^{2}(B(u))$, thereby proving the contrapositive of Theorem 3.1. The intuition behind the claim is explained in the course of the next few paragraphs, which should be regarded as a preparation for the proofs of Lemma 3.2 and Proposition 3.3. It is on these results that the proof of Theorem 3.1 ultimately rests.

3.1. Preparation for proof of Theorem 3.1. Suppose $v \neq 0$ satisfies (3.6) and (3.7). Applying Lemma 2.3 to (3.6), and bearing in mind that $\mathcal{L}^{2}(B)>0$, we see that

$$
\frac{4}{t(1-t)} \leq \frac{\delta}{\epsilon^{2}}
$$

where

$$
t=\frac{\mathcal{L}^{2}(B)}{\mathcal{L}^{1}(\Pi(v))}
$$


Note that this automatically implies $\delta \geq 16 \epsilon^{2}$, which is not a restriction since we already know from Proposition 2.5 that $u=0$ is a global minimizer of $E_{1}$ in $\mathcal{A}_{1}$ when $\delta<16 \epsilon^{2}$. Rearranging (3.8) gives

$$
\frac{4 \epsilon^{2}}{\delta} \leq \frac{\mathcal{L}^{2}(B)}{\mathcal{L}^{1}(\Pi(v))} \leq 1-\frac{4 \epsilon^{2}}{\delta}
$$

where, as usual, $B=B(v)$ for short. The upper bound is merely a slight improvement on the trivial inequality $t \leq 1$; the lower bound is new information on the set $B(v)$ and is a direct consequence of the assumption (3.6). Inequality (3.9) can be interpreted by supposing for the sake of argument that it applies to some rectangle $B$ whose sides are parallel to the coordinate axes. Its "height" would then be bounded below by a fixed constant. For more general sets $B$, condition (3.9) should be interpreted in an average sense, viz.

$$
\frac{1}{\mathcal{L}^{1}(\Pi(v))} \int_{\Pi(v)} \mathcal{L}^{1}\left(l_{x} \cap B\right) d x \geq \frac{4 \epsilon^{2}}{\delta}
$$

The following lemma establishes inequalities (3.11) and (3.13) that will be needed in this and subsequent sections of the paper. Part (b) involves all the terms appearing in the energy $E_{1}(v)$ and the $L^{2}$ norm of $v$; it should be regarded as the backbone of Theorem 3.1. Inequality (3.11) is used in section 4.2.

Lemma 3.2. Let $v \in \mathcal{A}_{1}^{+}$and let $B=B(v)$.

(a) Define the set

$$
\widehat{\Pi}=\{(x, y): 0 \leq y \leq 1, x \in \Pi(v)\}
$$

and let

$$
\rho=\frac{f_{\widehat{\Pi}} v^{2} \mathrm{dx}}{\int_{\Omega} v^{2} \mathrm{dx}}
$$

Then

$$
\int_{\Omega} v_{x}^{2} \mathrm{dx} \geq \rho \int_{\hat{\Pi}} v^{2} \mathrm{dx}
$$

(b) Let $M>0$ and define

$$
\begin{aligned}
\Pi_{M}(v) & =\left\{x \in \Pi(v): \int_{l_{x}} v_{y y}^{2}(x, y) d y<M\right\}, \\
B_{M} & =\left\{(x, y) \in B: \quad x \in \Pi_{M}(v)\right\} .
\end{aligned}
$$

Then there is a constant $C>0$ independent of $v$ and the dimensions of $\Omega$ such that

$$
\left(\int_{\Omega} v^{2}(x, y) \mathrm{dx}\right)^{\frac{1}{2}}\left(\int_{\Omega} v_{x}^{2}(x, y) \mathrm{dx}\right)^{\frac{1}{2}} \geq \frac{C}{M}\left(\frac{\mathcal{L}^{2}\left(B_{M}\right)}{\mathcal{L}^{1}\left(\Pi_{M}(v)\right)}\right)^{2} .
$$

Proof. By applying standard results from the theory of Sobolev functions, we may assume without loss of generality that $x \mapsto v^{2}(x, y)$ is weakly differentiable, and hence that

$$
\int_{0}^{x^{\prime}} v(x, y) v_{x}(x, y) d x=\frac{1}{2} v^{2}\left(x^{\prime}, y\right)
$$


on using the boundary condition $v(0, y)=0$. The left-hand side of this inequality is trivially bounded above by

$$
U\left(x^{\prime}, y\right):=\frac{\sigma^{2}}{2} \int_{0}^{x^{\prime}} v^{2}(x, y) d x+\frac{1}{2 \sigma^{2}} \int_{0}^{x^{\prime}} v_{x}^{2}(x, y) d x
$$

for all nonzero $\sigma$; the dependence on $\sigma$ will be minimized out later. The inequality

$$
v^{2}\left(x^{\prime}, y\right) \leq U\left(x^{\prime}, y\right)
$$

valid for $0 \leq x^{\prime} \leq L$ and $0 \leq y \leq 1$, can now be adapted to prove statements (a) and (b) as follows.

Proof of (a). We begin by integrating (3.15) over $\widehat{\Pi}$. Now

$$
\begin{aligned}
\int_{\Pi(v)} \int_{0}^{1} \int_{0}^{x^{\prime}} v^{2}(x, y) d x d y d x^{\prime} & \leq \int_{\Pi(v)}\left(\int_{0}^{L} \int_{0}^{1} v^{2}(x, y) d y d x\right) d x^{\prime} \\
& =\mathcal{L}^{1}(\Pi(v)) \int_{\Omega} v^{2} \mathrm{dx} .
\end{aligned}
$$

The term involving $v_{x}^{2}$ in (3.14) can be estimated in the same way. It follows that

$$
\int_{\Pi(v)} \int_{0}^{1} U\left(x^{\prime}, y\right) d y d x^{\prime} \leq \mathcal{L}^{1}(\Pi(v))\left(\frac{\sigma^{2}}{2} \int_{\Omega} v^{2}(x, y) \mathrm{dx}+\frac{1}{2 \sigma^{2}} \int_{\Omega} v_{x}^{2} \mathrm{dx}\right)
$$

and hence, in view of (3.15), that

$$
f_{\widehat{\Pi}} v^{2} \mathrm{dx} \leq\left(\int_{\Omega} v_{x}^{2} \mathrm{dx}\right)^{\frac{1}{2}}\left(\int_{\Omega} v^{2} \mathrm{dx}\right)^{\frac{1}{2}}
$$

Here, we have taken $\sigma^{2}=\left(\int_{\Omega} v_{x}^{2} \mathrm{dx}\right)^{\frac{1}{2}}\left(\int_{\Omega} v^{2} \mathrm{dx}\right)^{-\frac{1}{2}}$ in the right-hand side of inequality (3.16). Part (a) now follows by rearranging (3.17).

Proof of (b). Let $x^{\prime} \in \Pi_{M}(v)$ and $0 \leq y \leq 1$. By (3.3) we have

$$
\int_{l_{x^{\prime}}} v^{2} d y \geq \frac{C}{\int_{l_{x^{\prime}}} v_{y y}^{2} d y} \mathcal{L}^{1}\left(l_{x^{\prime}} \cap B\right)^{2}
$$

which in view of the fact that $x^{\prime} \in \Pi_{M}(v)$ implies

$$
\int_{l_{x^{\prime}}} v^{2} d y \geq \frac{C}{M} \mathcal{L}^{1}\left(l_{x^{\prime}} \cap B\right)^{2} .
$$

Integrating both sides of this expression over $\Pi_{M}(v)$ and applying Jensen's inequality to the right-hand side yields

$$
\int_{\Pi_{M}(v)} \int_{l_{x^{\prime}}} v^{2} d y d x^{\prime} \geq \frac{C}{M} \frac{\mathcal{L}^{2}\left(B_{M}\right)^{2}}{\mathcal{L}^{1}\left(\Pi_{M}(v)\right)} .
$$

The expression on the right is almost the desired lower bound; a factor of $\mathcal{L}^{1}\left(\Pi_{M}(v)\right)$ is missing from the denominator. But we know from (3.15) that

$$
\int_{\Pi_{M}(v)} \int_{0}^{1} U\left(x^{\prime}, y\right) d y d x^{\prime} \geq \int_{\Pi_{M}(v)} \int_{l_{x^{\prime}}} v^{2} d y d x^{\prime}
$$


and therefore by analogy with the argument leading to (3.16) we have

$$
\int_{\Pi_{M}(v)} \int_{0}^{1} U\left(x^{\prime}, y\right) d y d x^{\prime} \leq \mathcal{L}^{1}\left(\Pi_{M}(v)\right)\left(\frac{\sigma^{2}}{2} \int_{\Omega} v^{2}(x, y) \mathrm{dx}+\frac{1}{2 \sigma^{2}} \int_{\Omega} v_{x}^{2} \mathrm{dx}\right) .
$$

Putting this together with (3.18) and (3.19), and choosing $\sigma$ as in part (a), gives

$$
\left(\int_{\Omega} v_{x}^{2} \mathrm{dx}\right)^{\frac{1}{2}}\left(\int_{\Omega} v^{2} \mathrm{dx}\right)^{\frac{1}{2}} \geq \frac{C}{M}\left(\frac{\mathcal{L}^{2}\left(B_{M}\right)}{\mathcal{L}^{1}\left(\Pi_{M}(v)\right)}\right)^{2}
$$

concluding the proof.

We continue to suppose that $v \in \mathcal{A}_{1}^{+}$satisfies (3.6) and (3.7). By applying the definition of $\Pi_{M}(v)$,

$$
M \chi_{\Pi(v) \backslash \Pi_{M}(v)}(x) \leq \int_{l_{x}} v_{y y}^{2} d y
$$

from which it follows by integrating and then applying (3.7) that

$$
\mathcal{L}^{1}\left(\Pi(v) \backslash \Pi_{M}(v)\right) \leq \frac{\delta \epsilon^{-2} \mathcal{L}^{2}(B)}{M} .
$$

Hence

$$
\begin{aligned}
\mathcal{L}^{2}\left(B \backslash B_{M}\right) & =\int_{\Pi(v) \backslash \Pi_{M}(v)} \mathcal{L}^{1}\left(l_{x} \cap B\right) d x \\
& \leq \mathcal{L}^{1}\left(\Pi(v) \backslash \Pi_{M}(v)\right) \\
& \leq \frac{\delta \epsilon^{-2} \mathcal{L}^{2}(B)}{M}
\end{aligned}
$$

We are free to choose $M=2 \delta \epsilon^{-2}$, thereby ensuring

$$
\mathcal{L}^{2}\left(B_{M}\right) \geq \frac{1}{2} \mathcal{L}^{2}(B)
$$

Now we combine these observations, an upper bound on $\frac{\int_{\Omega} v_{x}^{2} \mathrm{dx}}{\mathcal{L}^{2}(B)}$, and Lemma 3.2 to give lower bounds on $\int_{\Omega} v^{2} \mathrm{dx}$ and $\mathcal{L}^{2}(B)$.

Proposition 3.3. Let $v \in \mathcal{A}_{1}^{+}$satisfy (3.6) and (3.7). Then there is a constant $C$ independent of $v$ and the dimensions of $\Omega$ such that

$$
\left(\int_{\Omega} v^{2} \mathrm{dx}\right)^{\frac{1}{2}} \geq \frac{C \epsilon^{6}}{\left(\mathcal{L}^{2}(B)\right)^{\frac{1}{2}} \delta^{\frac{7}{2}}} .
$$

Furthermore, provided $\frac{\delta}{\delta_{1}}$ is sufficiently large,

$$
\begin{aligned}
\left(\int_{\Omega} v^{2} \mathrm{dx}\right)^{\frac{1}{2}} & \geq C \epsilon^{\frac{7}{2}} \delta^{-2}, \\
\mathcal{L}^{2}(B(v)) & \geq C \epsilon^{6} \delta^{-4} L^{-1} .
\end{aligned}
$$


Proof. Applying Lemma 3.2 to $v$ with the choice of $M$ made above, and by using inequalities (3.7) and (3.9), we see that

$$
\begin{aligned}
\left(\int_{\Omega} v^{2} \mathrm{dx}\right)^{\frac{1}{2}} & \geq \frac{1}{\left(\int_{\Omega} v_{x}^{2}\right)^{\frac{1}{2}}} \frac{C}{\delta \epsilon^{-2}}\left(\frac{\mathcal{L}^{2}(B)}{\mathcal{L}^{1}(\Pi(u))}\right)^{2} \\
& \geq \frac{C \epsilon^{2}}{\delta}\left(\frac{\epsilon^{2}}{\delta}\right)^{2} \frac{1}{\left(\delta \mathcal{L}^{2}(B)\right)^{\frac{1}{2}}} \\
& =\frac{C \epsilon^{6}}{\left(\mathcal{L}^{2}(B)\right)^{\frac{1}{2}} \delta^{\frac{7}{2}}} .
\end{aligned}
$$

This inequality is (3.22). The constant $C$ changes from line to line but it remains independent of $\epsilon, \delta$, and $L$.

To prove $(3.23)$ we let $p:=\|v\|_{L^{2}(\Omega)}$ and $q:=\mathcal{L}^{2}(B)^{\frac{1}{2}}$ and note that (3.22) implies

$$
p q \geq C \epsilon^{6} \delta^{-\frac{7}{2}}
$$

For brevity we denote the right-hand side of this inequality by $f$.

Next, we use the simple interpolation inequality (2.10) together with (3.7) to get

$$
C \epsilon^{2} \delta \geq \frac{\mathcal{L}^{2}(B)}{\int_{\Omega} v^{2} \mathrm{dx}} .
$$

Hence,

$$
p \geq g q
$$

where $g=C \epsilon \delta^{-\frac{1}{2}}$.

Finally, (2.11) and (3.7) together imply

$$
\delta \mathcal{L}^{2}(B) \geq \frac{C}{L^{2}} \int_{\Omega} v^{2} \mathrm{dx},
$$

which in terms of $p$ and $q$ can be written

$$
p \leq C L \delta^{\frac{1}{2}} q .
$$

The aim is to determine the $(p, q)$ region which is compatible with these inequalities. This can be done by looking at Figure 2 . Note that the line with equation $p=C L \delta^{\frac{1}{2}} q$ lies above the line with equation $p=g q$, provided $\delta \geq \frac{C \epsilon}{L}$, which, in view of Theorem 3.1 , is true whenever $\frac{\delta}{\delta_{1}}$ is sufficiently large. This ensures that the required $(p, q)$ region is nonempty.

It is immediate that solving $p q=f$ and $p=g q$ yields the smallest possible value $p_{\min }$ of $p$ consistent with (3.6) and (3.7). The result is

$$
p_{\min }=C \epsilon^{\frac{7}{2}} \delta^{-2}
$$

giving the lower bound on $\|v\|_{L^{2}(\Omega)}$ stated in (3.23). Similarly, the smallest value $q_{\min }$ of $q$ consistent with (3.6) and (3.7) is found by solving for $q$ in $p q=f$ and $p=C L \delta^{\frac{1}{2}} q$. The result is

$$
q_{\min }=C \epsilon^{3} \delta^{-2} L^{-\frac{1}{2}}
$$




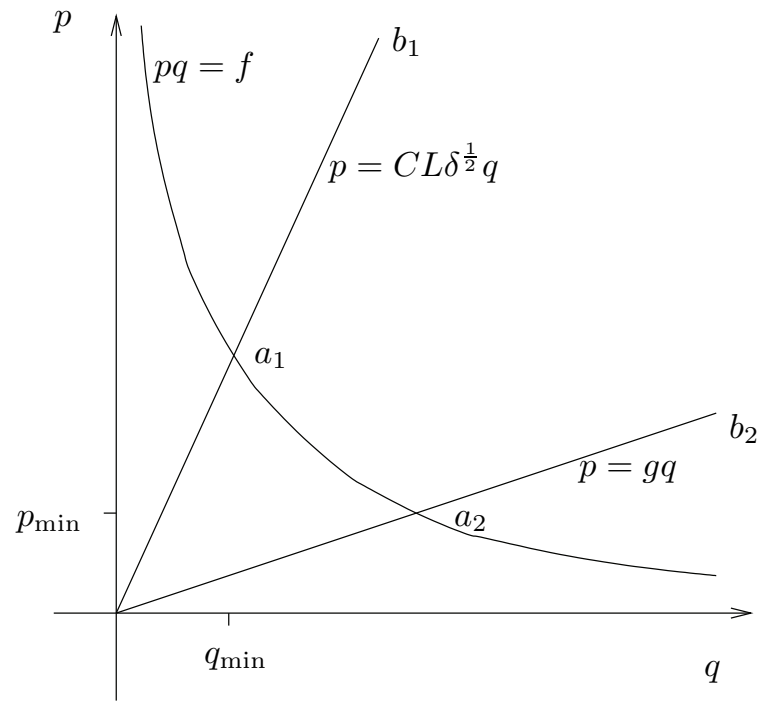

FIG. 2. The region compatible with (3.6) and (3.7) lies within the wedge formed by the lines $a_{1} b_{1}$ and $a_{2} b_{2}$ and above the curve containing the points $a_{1}$ and $a_{2}$.

giving the claimed lower bound on $\mathcal{L}^{2}(B)$. This concludes the proof of Proposition 3.3 .

We now draw the preceding results together.

Proof of Theorem 3.1. We prove the contrapositive under the assumption $v \neq 0$. Suppose $E_{1}(v) \leq E_{1}(0)$. Then in particular both

$$
\int_{\Omega} v_{y y}^{2} \mathrm{dx} \leq \frac{\delta}{\epsilon} \mathcal{L}^{2}(B)
$$

and

$$
\int_{\Omega} v_{x}^{2} \mathrm{dx} \leq \delta \mathcal{L}^{2}(B)
$$

must hold. These equations are (3.6) and (3.7), respectively, where, in view of $v \neq$ 0 , it can be assumed that $\mathcal{L}^{2}(B)>0$. It follows from Proposition 3.3 that both $\|v\|_{L^{2}(\Omega)} \geq r(\epsilon, \delta)$ and $\mathcal{L}^{2}(B) \geq s(\epsilon, \delta)$ must hold, concluding the proof of Theorem 3.1.

Remark 4. The lines $p=g q$ and $p=C L \delta^{\frac{1}{2}} q$ coincide when $\delta \sim \delta_{1}$ and their relative positions are reversed, provided $\frac{\delta}{\delta_{1}}$ is small enough. Under these circumstances the region compatible with all three inequalities (3.25), (3.26), and (3.27) becomes empty and the starting point for these calculations, namely, the inequality $E_{1}(v) \leq E_{1}(0)$, is contradicted. But this makes sense since for small $\frac{\delta}{\delta_{1}}$ it is the case that $E_{1}(v)>E_{1}(0)$ for all nonzero $v$.

Remark 5. If the lower bounds on $\|v\|_{L^{2}(\Omega)}$ and $\mathcal{L}^{2}(B)$ were optimal in a scaling sense, then as $\delta \rightarrow \delta_{1}$ we might expect $r(\epsilon, \delta) \rightarrow \infty$ and $s(\epsilon, \delta) \rightarrow \mathcal{L}^{2}(\Omega)$. In other words, the constraints on $\|v\|_{2}$ and $\mathcal{L}^{2}(B(v))$ should become redundant as $\delta$ approaches $\delta_{1}$, since when $\delta \sim \delta_{1}$ the state $u=0$ is the unique global minimizer and the desired inequality $E_{1}(v) \geq E_{1}(0)$ should hold for any admissible $v$. But it is easily checked that $\delta \rightarrow \delta_{1}$ implies neither $r(\epsilon, \delta) \rightarrow \infty$ nor $s(\epsilon, \delta) \rightarrow \mathcal{L}^{2}(\Omega)$. Thus $r(\epsilon, \delta)$ 
and $s(\epsilon, \delta)$ would appear to be smaller than they could be, i.e., suboptimal. Said differently, the inequalities (3.1) and (4.5) are likely to be sufficient but not necessary conditions for the inequality $E_{1}(v) \geq E_{1}(0)$.

4. The effect of modifying the surface energy term. Let us assume that $\frac{\delta}{\delta_{1}}$ is large enough to ensure that $u=0$ is not the global minimizer of $E_{i}$ for $i=1,2,3$. Theorem 2.2 assures us that this is possible, and that in this case the global minimizer $u_{1}$ satisfies $\mathcal{L}^{2}\left(B\left(u_{1}\right)\right)>0$. Thus in the energy landscape $\left\{E_{1}(v ; \delta): v \in \mathcal{A}_{1}\right\}$, for example, the points $u=0$ and $u=u_{1}$ are local, respectively, global, minimizers which may be separated by a so-called energy barrier. A natural way to measure such an energy barrier is to calculate $\mathcal{E}$, where

$$
\mathcal{E}=\inf \left\{\sup _{0 \leq t \leq 1}\left\{E_{1}^{\prime}(u(\cdot ; t)\}:(u(\cdot ; t))_{0 \leq t \leq 1} \text { joins } u=0 \text { to } u=u_{1} \text { in } \mathcal{A}_{1}\right\} .\right.
$$

Here, a path $(u(\cdot ; t))_{0 \leq t \leq 1} \subset \mathcal{A}_{1}$ joins $u=0$ to $u=u_{1}$, provided

(i) $u(\cdot ; 0)=0$,

(ii) $u(\cdot ; 1)=u_{1}$,

(iii) $\left|u_{y}(\cdot ; t)\right| \leq 1$ a.e. in $\Omega$ and for $0 \leq t \leq 1$,

(iv) $\mathcal{L}^{2}(B(u(\cdot ; t)))$ is continuous as a function of $t$ in a right-neighborhood of each $t_{0}$ such that $\mathcal{L}^{2}\left(B\left(u\left(\cdot, t_{0}\right)\right)\right)=0$.

Clearly, other interpretations are possible. Ours is motivated by the simple observation that in any "physically reasonable" path $(u(\cdot ; t))_{0 \leq t \leq 1}$ in $\mathcal{A}_{1}$ connecting $u=0$ to $u=u_{1}$, where $\mathcal{L}^{2}\left(B\left(u_{1}\right)\right)>0$, it should not be possible for $\mathcal{L}^{2}(B(u(\cdot, t)))$ to jump from 0 to $\mathcal{L}^{2}\left(B\left(u_{1}\right)\right)>0$. Property (iv) ensures that this cannot happen. The restriction $\left|u_{y}(\cdot ; t)\right| \leq 1$ a.e. in $\Omega$ is analytically convenient.

Section 4 of this paper focuses on deciding whether or not the quantity $\mathcal{E}$ is strictly positive. The question is addressed by examining the behavior of $E_{1}^{\prime}(v)=$ $E_{1}(v)-E_{1}(0)$, in particular for those $v \in \mathcal{A}_{1}^{+}$satisfying $\left|v_{y}\right| \leq 1$ a.e. with $\mathcal{L}^{2}(B(v))$ small and positive. The results can be found in Theorem 4.1 below, all of which are supportive of the existence of an energy barrier, that is, of $\mathcal{E}>0$. They do not, however, quantify $\mathcal{E}$ in terms of the parameters $\epsilon, \delta, L$ appearing in the problem. In the following we use the notation $|\Pi(v)|=\mathcal{L}^{1}(\Pi(v))$.

TheOREM 4.1. Let $v \in \mathcal{A}_{1}$ and let $\frac{\delta}{\delta_{1}}$ be large enough to ensure that $u=0$ is not the global minimizer of $E_{1}$ in $\mathcal{A}$. Then we have the following:

(a) For any $v \in \mathcal{A}_{1}^{+}$with $\left|v_{y}\right| \leq 1$ a.e. and such that diam supp $v \rightarrow 0$ as $\mathcal{L}^{2}(B(v)) \rightarrow 0$,

$$
\liminf _{|\Pi(v)| \rightarrow 0} \frac{E_{1}^{\prime}(v)}{|\Pi(v)|}=+\infty
$$

where $\Pi(v)$ is the projection of $B(v)$ onto the $x$-axis, and $|\Pi(v)|=\mathcal{L}^{1}(\Pi(v))$.

(b) For any $\sigma \in\left(\frac{1}{2}, 1\right)$ and $v \in \mathcal{A}_{1}^{+}$with $\left|v_{y}\right| \leq 1$ a.e.,

$$
\liminf _{|\Pi(v)| \rightarrow 0} \frac{E_{1}^{\prime}(v)}{|\Pi(v)|^{\sigma+1}}=+\infty .
$$

(c) Moreover, there is a sequence $v^{(j)}$ in $\mathcal{A}_{1}$ such that

$$
E_{1}^{\prime}\left(v^{(j)}\right) \rightarrow 0+
$$

as $j \rightarrow \infty$ and with $\mathcal{L}^{2}\left(B\left(v^{(j)}\right)\right)>0$ for all $j$. The sequence $v^{(j)}$ can be assumed to satisfy $\left|v_{y}^{(j)}\right| \leq 1$ a.e. in $\Omega$. 
We prove parts (a) and (b) of Theorem 4.1 in section 4.1 below, while the construction of sequences $v^{(j)}$ satisfying part (c) is given in Proposition 4.3 below. An improved version of part (c) of Theorem 4.1 is given in Theorem 4.5. It happens that functions $v$ satisfying part (c) of the theorem can be constructed directly in the case of the functional $E_{1}$; see Proposition 4.3 below. The construction of the relevant $v$ in the cases of $E_{2}$ and $E_{3}$ is indirect and more involved; see section 4.3 below.

To infer that $\mathcal{E}>0$ from Theorem 4.1 it is clearly enough to note that the prefactor $\frac{c^{\frac{7}{3}} \epsilon^{\frac{7}{3}} k^{\frac{1}{3}}}{C^{\frac{1}{3}}} M$ in (4.17) is independent of $|\Pi|$ as $|\Pi| \rightarrow 0$. Since we have already observed that passing from the austenite state $u=0$ to the global minimizer $u=u_{1}$ necessarily involves changing $\Pi$ from a null set to a nonnull set, and taking into account our definition of path joining $u=0$ to $u=u_{1}$, it follows that $\mathcal{E}$ must be strictly positive.

We recall that the inequalities $E_{3}^{\prime}(v) \geq E_{2}^{\prime}(v) \geq E_{2}^{\prime}(v)$ hold for all suitable $v$. In particular, they imply that parts (a) and (b) also hold for the functionals $E_{3}^{\prime}$ and $E_{2}^{\prime}$.

4.1. A lower bound on the growth of $\boldsymbol{E}_{1}(v)-\boldsymbol{E}_{1}(\mathbf{0})$. Let $v \in \mathcal{A}_{1}$, and as before let

$$
\widehat{\Pi}(v)=\Pi(v) \times[0,1] .
$$

The following estimate will be needed in the course of Theorem 4.1. The proof is elementary and only the main steps are indicated.

Proposition 4.2. Let $v:[0,1] \rightarrow \mathbb{R}$ be $W^{2,2}((0,1)) \cap C^{1}([0,1])$ and suppose $v(0)=v(1)$. Suppose also that $\left|v_{y}(y)\right| \leq 1$ for all $y \in[0,1]$. Let $\alpha$ be an open subinterval of $(0,1)$ for which the following conditions hold:

(i) $v_{y}$ is monotone and obeys $\left|v_{y}\right|<1$ on $\alpha$;

(ii) $\alpha$ has an endpoint in common with a subinterval $\gamma$ of $[0,1]$ such that $\left|v_{y}(y)\right|=$ 1 for all $y \in \gamma$, and $|\gamma|>0$;

(iii) the quantity

$$
\beta:=o s c_{\alpha} v_{y}
$$

satisfies $\beta \leq 1$.

Define the real number $\Lambda(\alpha)$ by

$$
\frac{\beta^{2}}{\Lambda(\alpha)}:=\int_{\alpha} v_{y y}^{2}(y) d y
$$

Then

$$
\int_{\alpha} v^{2} d y \geq k^{2} \Lambda(\alpha)\left(\Lambda(\alpha)+\frac{|\gamma|}{2}\right)^{2},
$$

where $|\gamma|=\mathcal{L}^{1}(\gamma)$ and $k$ is a constant independent of $v$.

Proof. Without loss of generality, we can suppose $\gamma=\left[0,2 y_{0}\right]$ with $v\left(y_{0}\right)=0$, $v\left(2 y_{0}\right)=y_{0}$, and that $v_{y}$ is monotone decreasing on the interval $\alpha=\left[2 y_{0}, y_{1}\right]$ to $v_{y}\left(y_{1}\right)=(1-\beta)$. Let $h=v\left(y_{1}\right)-v\left(2 y_{0}\right)$ and let $l=|\alpha|$. If $h \geq\left(1-\frac{\beta}{2}\right) l$, then on $\left[y_{0}, y_{1}\right]$ the function $|v|$ is bounded below by the nonnegative piecewise affine function $p$, where

$$
p(y)= \begin{cases}y-y_{0} & \text { if } y_{0} \leq y \leq 2 y_{0} \\ y_{0}\left(\frac{y_{1}-y}{y_{1}-2 y_{0}}\right)+\left(y_{0}+l\left(1-\frac{\beta}{2}\right)\right)\left(\frac{y-2 y_{0}}{y_{1}-2 y_{0}}\right) & \text { if } 2 y_{0} \leq y \leq y_{1} .\end{cases}
$$


A calculation using $\int_{\alpha} v^{2}(y) d y \geq \int_{\alpha} p^{2}(y) d y$ shows that

$$
\int_{\alpha} v^{2} d y \geq \frac{1}{12} l\left(l+y_{0}\right)^{2} .
$$

It is straightforward to show that

$$
\int_{\alpha} v_{y y}^{2} d y \geq \frac{\beta^{2}}{l}
$$

which, in view of the definition of $\Lambda(\alpha)$ above, yields $l \geq \Lambda(\alpha)$. Substituting this, together with $y_{0}=\frac{1}{2}|\gamma|$ and $k^{2}=\frac{1}{12}$, into (4.4) verifies the estimate (4.3) in the case $h \geq\left(1-\frac{\beta}{2}\right) l$. If $h<\left(1-\frac{\beta}{2}\right) l$, then $\tau:=\frac{2}{\beta}(h-l(1-\beta))$ satisfies $\tau<l$, and it can be checked that

$$
\int_{\alpha} v_{y y}^{2} d y \geq \frac{\beta^{2}}{\tau}
$$

for all functions $v$ satisfying properties (i)-(iii) above. The definition of $\Lambda(\alpha)$ implies $\tau>\Lambda(\alpha)$, whence $h \geq \frac{1}{2} \Lambda(\alpha)$. The function $|v|$ is bounded below by the piecewise affine function

$$
\bar{p}(y)= \begin{cases}y-y_{0} & \text { if } y_{0} \leq y \leq 2 y_{0} \\ y_{0}\left(\frac{y_{1}-y}{y_{1}-2 y_{0}}\right)+\left(y_{0}+\frac{1}{2} \Lambda(\alpha)\right)\left(\frac{y-2 y_{0}}{y_{1}-2 y_{0}}\right) & \text { if } 2 y_{0} \leq y \leq y_{1}\end{cases}
$$

which can be used as before to confirm estimate (4.3) with the same choice of $k^{2}$ as above.

Proof of Theorem 4.1. Let $v \in \mathcal{A}_{1}^{+}$satisfy $\left|v_{y}\right| \leq 1$ a.e. in $\Omega$. By an approximation argument, we can suppose that $v(x, \cdot)$ satisfies the hypotheses of Proposition 4.2 for a.e. $x$ in $\Pi(v)$. Let $0<c<1$ be a fixed constant. For each $0 \leq x \leq L$ let

$$
S(x)=\bigcup_{j=1}^{N(x)} \alpha_{j}
$$

be the union of those intervals $\alpha_{j}$ satisfying hypotheses (i)-(iii) of Proposition 4.2 and for which, in addition, the corresponding $\beta_{j}$ satisfy $\beta_{j} \geq c$. The 1-periodicity of $v(x, \cdot)$ assures us that for a.e. $x \in \Pi(v)$ there is at least one interval $\alpha_{j}$ over which $v_{y}$ changes by at least $c$. Thus the union $S(x)$ is nonempty for a.e. $x \in \Pi(v)$. Henceforth, we abbreviate $\Pi(v)$ to $\Pi$ and define the set $S$ by

$$
S:=\{\{x\} \times S(x): x \in \Pi\} .
$$

Now, by Lemma 3.2,

$$
\int_{\Omega} v_{x}^{2} \mathrm{dx} \geq \frac{\rho}{|\Pi|} \int_{\widehat{\Pi}} v^{2} \mathrm{dx}
$$

Since $\widehat{\Pi} \supset S$, it follows from Proposition 4.2 above that

$$
\int_{\Omega} v_{x}^{2} \mathrm{dx} \geq \frac{\rho k^{2}}{|\Pi|} \int_{\Pi} \sum_{j=1}^{N(x)} \Lambda\left(\alpha_{j}\right)\left(\Lambda\left(\alpha_{j}\right)+\frac{1}{2}\left|\gamma_{j}\right|\right)^{2} d x .
$$


For later use we recall that

$$
\rho=\frac{1}{|\Pi|} \frac{\int_{\widehat{\Pi}} v^{2} \mathrm{dx}}{\int_{\Omega} v^{2} \mathrm{dx}}
$$

Using the definition of $\Lambda\left(\alpha_{j}\right)$ given above, together with the condition $\beta_{j} \geq c$, yields

$$
\int_{\Omega} v_{y y}^{2} \mathrm{dx} \geq \int_{\Pi} \sum_{j=1}^{N(x)} \frac{c^{2}}{\Lambda\left(\alpha_{j}\right)} d x
$$

Let $F(v)=\int_{\Omega} \epsilon^{2} v_{y y}^{2}+v_{x}^{2} \mathrm{dx}$. Then

$$
F(v) \geq \int_{\Pi} c^{2} \epsilon^{2} \sum_{j=1}^{N(x)} \frac{1}{\Lambda\left(\alpha_{j}\right)}+\frac{k^{2} \rho}{|\Pi|} \sum_{j=1}^{N(x)} \Lambda\left(\alpha_{j}\right)\left(\Lambda\left(\alpha_{j}\right)+\frac{1}{2}\left|\gamma_{j}\right|\right)^{2} d x .
$$

Let $\bar{\Lambda}(x)=\sum_{j=1}^{N(x)} \Lambda\left(\alpha_{j}\right)$ and define $d(x)=\frac{\bar{\Lambda}(x)}{N(x)}$; note that $d(x)$ is the average length of the intervals $\alpha_{j}$ contained in $l_{x}$ and satisfying the hypotheses above. By writing

$$
c^{2} \epsilon^{2} \sum_{j=1}^{N(x)} \frac{1}{\Lambda\left(\alpha_{j}\right)}=c^{2} \epsilon^{2} N(x) \sum_{j=1}^{N(x)} \frac{1}{N(x)} f\left(\Lambda\left(\alpha_{j}\right)\right),
$$

where $f(s)=\frac{1}{s}$ is convex in $s>0$, we see that

$$
c^{2} \epsilon^{2} \sum_{j=1}^{N(x)} \frac{1}{\Lambda\left(\alpha_{j}\right)} \geq \frac{c^{2} \epsilon^{2} N(x)}{d(x)} .
$$

Also, since

$$
\sum_{j=1}^{N(x)} \Lambda\left(\alpha_{j}\right)\left(\Lambda\left(\alpha_{j}\right)+\frac{1}{2}\left|\gamma_{j}\right|\right)^{2}=\bar{\Lambda}(x) \sum_{j=1}^{N(x)} \frac{\Lambda\left(\alpha_{j}\right)}{\bar{\Lambda}(x)} g\left(\Lambda\left(\alpha_{j}\right)+\frac{1}{2}\left|\gamma_{j}\right|\right),
$$

where $g(t)=t^{2}$ is convex in $t$, it follows that

$$
\sum_{j=1}^{N(x)} \Lambda\left(\alpha_{j}\right)\left(\Lambda\left(\alpha_{j}\right)+\frac{1}{2}\left|\gamma_{j}\right|\right)^{2} \geq \bar{\Lambda}(x) g\left(\sum_{j=1}^{N(x)} \frac{\Lambda\left(\alpha_{j}\right)}{\bar{\Lambda}(x)}\left(\Lambda\left(\alpha_{j}\right)+\frac{1}{2}\left|\gamma_{j}\right|\right)\right) .
$$

But

$$
\bar{\Lambda}(x) g\left(\sum_{j=1}^{N(x)} \frac{\Lambda\left(\alpha_{j}\right)}{\bar{\Lambda}(x)}\left(\Lambda\left(\alpha_{j}\right)+\frac{1}{2}\left|\gamma_{j}\right|\right)\right)=\frac{d(x)}{N(x)}\left(\sum_{j=1}^{N(x)} \frac{\Lambda\left(\alpha_{j}\right)}{d(x)}\left(\Lambda\left(\alpha_{j}\right)+\frac{1}{2}\left|\gamma_{j}\right|\right)\right)^{2},
$$

and hence

$$
F(v) \geq \int_{\Pi} \frac{c^{2} \epsilon^{2} N(x)}{d(x)}+\frac{k^{2} \rho d(x)}{|\Pi| N(x)}\left(\sum_{j=1}^{N(x)} \frac{\Lambda\left(\alpha_{j}\right)}{d(x)}\left(\Lambda\left(\alpha_{j}\right)+\frac{1}{2}\left|\gamma_{j}\right|\right)\right)^{2} d x .
$$


Let

$$
W^{2}(x)=\left(\sum_{j=1}^{N(x)} \frac{\Lambda\left(\alpha_{j}\right)}{d(x)}\left(\Lambda\left(\alpha_{j}\right)+\frac{1}{2}\left|\gamma_{j}\right|\right)\right)^{2}
$$

and note that the integrand on the right-hand side of (4.9) is pointwise bounded below by $\frac{2 c \epsilon k \rho^{\frac{1}{2}} W(x)}{|\Pi|^{\frac{1}{2}}}$. Thus

$$
F(v) \geq \int_{\Pi} \frac{2 c \epsilon k \rho^{\frac{1}{2}} W(x)}{|\Pi|^{\frac{1}{2}}} d x
$$

Since $f$ and $g$ above are strictly convex, inequalities (4.7) and (4.8) become equalities if and only if $\Lambda\left(\alpha_{j}\right)=\Lambda(x), j=1, \ldots, N(x)$, and $\left|\gamma_{j}\right|=|\gamma|, j=1, \ldots, N(x)$, for some $\Lambda(x)$ and $|\gamma|$. In these circumstances $d(x)=\Lambda(x)$, and $W(x)=N(x)(\Lambda(x)+$ $\left.\frac{1}{2}|\gamma|\right)$. Furthermore, the inequality

$$
\frac{c^{2} \epsilon^{2} N(x)}{d(x)}+\frac{\rho k^{2} d(x) W^{2}(x)}{|\Pi| N(x)} \geq \frac{2 c \epsilon k \rho^{\frac{1}{2}} W(x)}{|\Pi|^{\frac{1}{2}}}
$$

becomes an equality if and only if

$$
\frac{c \epsilon N(x)}{d(x)}=\frac{\rho^{\frac{1}{2}} k W(x)}{|\Pi|^{\frac{1}{2}}},
$$

which when equality holds in (4.7) and (4.8) further implies

$$
\rho^{\frac{1}{2}} \Lambda(x)\left(\Lambda(x)+\frac{1}{2}|\gamma|\right)=\frac{\epsilon c|\Pi|^{\frac{1}{2}}}{k} .
$$

Proof of part (a). From (4.10) and (4.12),

$$
F(v) \geq \int_{\Pi} 2 c^{2} \epsilon^{2} \frac{N(x)}{\Lambda(x)} d x .
$$

Therefore $\frac{E_{1}^{\prime}(v)}{|\Pi|} \geq \int_{\Pi} \frac{2 \epsilon^{2} c^{2} N(x)}{\Lambda(x)|\Pi|} d x-\delta$, where the inequality $\mathcal{L}^{2}(B(v)) \leq|\Pi|$ has been used. By definition, $\Lambda(x)$ is the vertical distance over which $v_{y}(x, \cdot)$ changes by at least $c$; in particular, $\Lambda(x) \leq \operatorname{diam} \operatorname{supp} v$ for a.e. $x \in \Pi$. Hence

$$
\frac{E_{1}^{\prime}(v)}{|\Pi|} \geq \frac{2 \epsilon^{2} c^{2}}{\operatorname{diam} \operatorname{supp} v}-\delta,
$$

with $c$ independent of diam $\operatorname{supp} v$. Statement (a) of the theorem is now immediate.

Proof of part (b). The argument proceeds in two steps.

Step 1 . We will obtain a lower bound on $\rho^{\frac{1}{2}}$ using (4.12), (4.6) and the estimate (4.3) above as follows. We may assume $v$ is such that $\Lambda\left(\alpha_{j}\right)=\Lambda(x), j=1, \ldots, N(x)$, and $\left|\gamma_{j}\right|=|\gamma|, j=1, \ldots, N(x)$, for some $\Lambda(x)$ and $|\gamma|$, thereby ensuring that $F(v)$ is minimized and that consequently (4.12) holds. From (4.3), and with the shorthand 
notation $\|v\|_{2}=\|v\|_{2, \Omega}$,

$$
\begin{aligned}
\rho\|v\|_{2}^{2} & \geq \frac{k^{2}}{|\Pi|} \int_{\Pi} N(x) \Lambda(x)\left(\Lambda(x)+\frac{1}{2}|\gamma|\right)^{2} d x \\
& \geq \frac{k^{2}}{|\Pi|} \int_{\Pi} \Lambda(x)\left(\Lambda(x)+\frac{1}{2}|\gamma|\right) W(x) d x \\
& \geq \frac{k^{2}}{|\Pi|} \int_{\Pi} \frac{c \epsilon}{k} \rho^{-\frac{1}{2}}|\Pi|^{\frac{1}{2}} N(x)\left(\Lambda(x)+\frac{1}{2}|\gamma|\right) d x,
\end{aligned}
$$

where we have used (4.12) to obtain the last line above. Again using $W(x)=N(x)(\Lambda+$ $\left.\frac{1}{2}|\gamma|\right)$, it follows that

$$
\rho^{\frac{3}{2}}\|v\|_{2}^{2} \geq c \epsilon k|\Pi|^{\frac{1}{2}} f_{\Pi} W(x) d x
$$

giving

$$
\rho^{\frac{1}{2}} \geq\|v\|_{2}^{-\frac{2}{3}}(c \epsilon k)^{\frac{1}{3}}|\Pi|^{\frac{1}{6}}\left(f_{\Pi} W(x) d x\right)^{\frac{1}{3}} .
$$

Substituting this into (4.6) yields

$$
F(v) \geq 2(c \epsilon k)^{\frac{4}{3}}\|v\|_{2}^{-\frac{2}{3}}|\Pi|^{\frac{1}{3}}\left(f_{\Pi} W(x) d x\right)^{\frac{4}{3}} .
$$

Applying Jensen's inequality to the right-hand side further implies

$$
F(v) \geq 2(c \epsilon k)^{\frac{4}{3}}\|v\|_{2}^{-\frac{2}{3}}|\Pi|^{-1}\left(\int_{\Pi} W(x) d x\right)^{\frac{4}{3}} .
$$

The aim now is to replace the $\|v\|_{2}$ term in this last expression by a term involving only $|\Pi|, \rho$, and $W$. Now, by definition,

$$
\rho|\Pi|\|v\|_{2}^{2}=\int_{\widehat{\Pi}} v^{2} \mathrm{dx} .
$$

The assumptions on $v$ ensure that $\int_{\widehat{\Pi}} v^{2} \mathrm{dx} \leq C_{1} \int_{S} v^{2} \mathrm{dx}$ for some constant $C_{1}$, where $S$ is the set defined in (3.2), and that the estimate (4.3) holds with approximate equality, that is,

$$
\int_{S} v^{2} \mathrm{dx} \leq C_{2} \int_{\Pi} N(x) \Lambda(x)\left(\Lambda(x)+\frac{1}{2}|\gamma|\right)^{2}
$$

for some constant $C_{2}$ depending only on the domain parameters. The trivial bound $\Lambda(x)\left(\lambda(x)+\frac{1}{2}|\gamma|\right) \leq 1$ therefore gives

$$
\rho|\Pi|\|v\|_{2}^{2} \leq C_{1} C_{2} \int_{\Pi} W(x) d x .
$$

In particular,

$$
\|v\|_{2}^{-\frac{2}{3}} \geq\left(C \int_{\Pi} W(x) d x\right)^{-\frac{1}{3}}(\rho|\Pi|)^{\frac{1}{3}}
$$


where $C=C_{1} C_{2}$. Substituting this into (4.13) yields

$$
F(v) \geq \frac{2(c \epsilon k)^{\frac{4}{3}}}{C^{\frac{1}{3}}}(\rho|\Pi|)^{\frac{1}{3}} f_{\Pi} W(x) d x .
$$

The assumptions on $v$ imply that $\left|l_{x} \cap B(v)\right| \leq C_{3} N(x)|\gamma|$ for some constant $C_{3}$. Since $\mathcal{L}^{2}(B(v))=\int_{\Pi}\left|l_{x} \cap B(v)\right| d x$, it follows that $\mathcal{L}^{2}(B(v)) \leq C_{3} \int_{\Pi} W(x) d x$, where we have used the expression $W(x)=N(x)\left(\Lambda(x)+\frac{1}{2}|\gamma|\right)$. Recalling that $E_{1}^{\prime}(v)=$ $F(v)-\delta \mathcal{L}^{2}(B(v))$, we have

$$
E_{1}^{\prime}(v) \geq\left(\frac{2(c \epsilon k)^{\frac{4}{3}}}{|\Pi| C^{\frac{1}{3}}}(\rho|\Pi|)^{\frac{1}{3}}-C_{3} \delta\right) \int_{\Pi} W(x) d x .
$$

By (4.11),

$$
\int_{\Pi} W(x) d x \geq \int_{\Pi} \frac{c \epsilon N(x)|\Pi|^{\frac{1}{2}}}{\Lambda(x) k \rho^{\frac{1}{2}}} d x
$$

Inserting this into (4.15) and rearranging gives

$$
E_{1}^{\prime}(v) \geq\left(\frac{2(c \epsilon k)^{\frac{4}{3}}}{C^{\frac{1}{3}}}(\rho|\Pi|)^{\frac{1}{3}}-C_{3} \delta|\Pi|\right) \frac{c \epsilon}{k(\rho|\Pi|)^{\frac{1}{2}}} \int_{\Pi} \frac{N(x)}{\alpha(x)} d x .
$$

Since $\rho|\Pi| \leq 1, N(x) \geq 1$ and $\Lambda(x) \leq 1$ a.e. $x \in \Pi$, it follows that

$$
\frac{c \epsilon}{k(\rho \Pi)^{\frac{1}{2}}} \int_{\Pi} \frac{N(x)}{\Lambda(x)} d x \geq \frac{c \epsilon|\Pi|}{k} .
$$

Finally,

$$
E_{1}^{\prime}(v) \geq\left(\frac{2(c \epsilon k)^{\frac{4}{3}}}{C^{\frac{1}{3}}}(\rho|\Pi|)^{\frac{1}{3}}-C_{3} \delta|\Pi|\right) \frac{c \epsilon|\Pi|}{k} .
$$

In Step 2 below we compare the behaviors of $(\rho|\Pi|)^{\frac{1}{3}}$ and $|\Pi|$ as $|\Pi| \rightarrow 0$.

Step 2. We claim that if $\sigma>\frac{1}{2}$, then

$$
\liminf _{|\Pi| \rightarrow 0} \frac{(\rho|\Pi|)^{\frac{1}{3}}}{|\Pi|^{\sigma}}=+\infty
$$

Assume the claim is false for a contradiction. Then there exists $M>0$ such that

$$
(\rho|\Pi|)^{\frac{1}{3}} \leq M^{\frac{1}{3}}|\Pi|^{\sigma}
$$

for all sufficiently small $|\Pi|$. In view of Poincaré's inequality (2.11), we may without loss of generality assume that $\int_{\Omega} v^{2} \leq 1$. This implies in particular that $\rho|\Pi| \geq$ $\int_{S} v^{2} \mathrm{dx}$ and hence, with the aid of (4.3) and (4.12), that

$$
\rho|\Pi| \geq \int_{\Pi} \frac{N(x)}{\Lambda(x)}\left(\frac{\epsilon c}{k}\right)^{2} \frac{|\Pi|}{\rho} d x
$$


Gathering terms in $\rho$ on the left-hand side and applying (4.1), together with the simple estimates $\Lambda(x) \leq 1 \leq N(x)$, yields

$$
|\Pi|^{6 \sigma-3} \geq\left(\frac{\epsilon c}{M k}\right)^{2} .
$$

To finish the proof of the claim it suffices to note that, since $\sigma>\frac{1}{2}$, the left-hand side converges to zero as $|\Pi| \rightarrow 0$, while the right-hand side is bounded below independently of $|\Pi|$.

In order to conclude the proof of statement (b) of Theorem 4.1 we apply the above claim to (4.16) as follows. Let $M>0$ and fix $\sigma \in\left(\frac{1}{2}, 1\right)$. By the claim, there exists $\eta=\eta(M, \sigma)>0$ such that if $|\Pi|<\eta$, then

$$
E_{1}^{\prime}(v) \geq\left(\frac{2(c \epsilon k)^{\frac{4}{3}}}{C^{\frac{1}{3}}} M|\Pi|^{\sigma}-C_{3} \delta|\Pi|\right) \frac{c \epsilon|\Pi|}{k} .
$$

In particular,

$$
E_{1}^{\prime}(v) \geq \frac{c^{\frac{7}{3}} \epsilon^{\frac{7}{3}} k^{\frac{1}{3}}}{C^{\frac{1}{3}}} M|\Pi|^{\sigma+1}
$$

provided $|\Pi|$ is sufficiently small. Since $M$ is arbitrary, this proves statement (b).

4.2. Constructing $v$ such that $E_{1}^{\prime}(v) \rightarrow 0$ as $\mathcal{L}^{2}(B(v)) \rightarrow 0$.

Proposition 4.3. Let $\frac{\delta}{\delta_{1}}$ be large enough to ensure that $u=0$ is not the global minimizer of $E_{1}$ in $\mathcal{A}_{1}$. Then

$$
\liminf _{\mu \rightarrow 0} \inf \left\{E_{1}(v): v \in \mathcal{A}_{1}^{+} \text {such that } \mathcal{L}^{2}(B(v))<\mu\right\}=0 .
$$

In other words, sets $B(v)$ of positive measure can be introduced at arbitrarily small energies, as measured by $E_{1}$. Moreover, (4.18) also holds if the functions $v$ are required to satisfy $\left|v_{y}\right| \leq 1$ a.e. in $\Omega$.

Proof. The proof of (4.18) is by construction as follows. Let $a, d>0, \lambda>1$, $f(x)=\lambda\left(\frac{x-(L-d)}{d}\right)^{2}$ and define $v:[L-d, L] \times[0,2 a] \rightarrow \mathbb{R}$ by

$$
v(x, y)= \begin{cases}\frac{f(x) y^{2}}{2 a} & \text { if } L-d \leq x \leq L, 0 \leq y \leq a, \\ a f(x)-\frac{f(x)(2 a-y)^{2}}{2 a} & \text { if } L-\delta \leq x \leq L, a \leq y \leq 2 a .\end{cases}
$$

Extend $v$ to $[L-d, L] \times[0,4 a]$ by reflection in $y=2 a$, i.e., $v(x, y):=v(x, 4 a-y)$ if $2 a \leq y \leq 4 a$. Finally, extend $v$ by zero outside $[L-d, L] \times[0,4 a]$. It can then be checked that

$$
F(v)=\lambda^{2}\left(C_{1} \frac{\epsilon^{2} d}{a}+C_{2} \frac{a^{3}}{d}\right)
$$

where $C_{1}$ and $C_{2}$ are constants independent of $a, d, \epsilon, \lambda$, and $L$, and where $F(v)=$ $\int_{\Omega} \epsilon^{2} v_{y y}^{2}+v_{x}^{2} \mathrm{dx}$. It is clear that $\mathcal{L}^{2}(B(v))>0$ for each positive $d$ and $a$ and for each $\lambda>1$. In fact,

$$
\mathcal{L}^{2}(B(v))=4 a d\left(1-\lambda^{-\frac{1}{2}}\right)^{2}
$$

and

$$
\mathcal{L}^{1}(\Pi(v))=d\left(1-\lambda^{-\frac{1}{2}}\right)
$$


Therefore for any given $\mu>0$ we can, by choosing $\lambda-1$ sufficiently small and positive, ensure that $\mu>\mathcal{L}^{2}(B(v))>0$ independently of the choice of $a$ and $\delta$. Taking $a^{2}=\left(\frac{C_{2}}{C_{1}}\right)^{\frac{1}{2}} \epsilon d$, and thereby minimizing $F(v)$ with respect to the parameters $a$ and $d$, we see that

$$
F(v)=2 \lambda^{2} \epsilon^{\frac{3}{2}} C_{1}^{\frac{1}{4}} C_{2}^{\frac{3}{4}} d^{\frac{1}{2}}
$$

Hence,

$$
E_{1}^{\prime}(v)=2 \lambda^{2} \epsilon^{\frac{3}{2}} C_{1}^{\frac{1}{4}} C_{2}^{\frac{3}{4}} d^{\frac{1}{2}}-4 \epsilon^{\frac{1}{2}}\left(\frac{C_{2}}{C_{1}}\right)^{\frac{1}{4}} \delta d^{\frac{3}{2}}\left(1-\lambda^{-\frac{1}{2}}\right)^{2}
$$

The first conclusion of the proposition now follows by letting $d \rightarrow 0$.

We modify the $v$ constructed above in order to confirm the last statement of the proposition as follows. First, the nonaffine part of the boundary of the set $B(v)$, where $v$ is defined in (4.2), is given by

$$
\Gamma(v):=\{(x, y) \in \Omega: f(x) y=a \text { or } f(x)(2 a-y)=a\}
$$

Let $y_{1}(x)$ and $y_{2}(x)$ satisfy $y_{1}(x) f(x)=1$ and $f(x)\left(2 a-y_{2}(x)\right)=a$, respectively. For each $x \in[L-d, L]$ and $y_{1}(x) \leq y \leq y_{2}(x)$ let $V(x, y)$ be the linear interpolant between $v\left(x, y_{1}(x)\right)$ and $v\left(x, y_{2}(x)\right)$, i.e.,

$$
V(x, y)=\left(\frac{y_{2}(x)-y}{y_{2}(x)-y_{1}(x)}\right) v\left(x, y_{1}(x)\right)+\left(\frac{y-y_{1}(x)}{y_{2}(x)-y_{1}(x)}\right) v\left(x, y_{2}(x)\right)
$$

It can be checked that $V_{y}=1$ for the specified ranges of $x$ and $y$. For $(x, y) \in$ $[L-d, L] \times[0,2 a]$ define

$$
\hat{v}(x, y):= \begin{cases}V(x, y) & \text { if }(x, y) \in B(v) \\ v(x, y) & \text { if }(x, y) \notin B(v)\end{cases}
$$

Then $\hat{v}$ satisfies $\hat{v}_{y}=1$ on $B(v)$, and $\hat{v}_{y}<1$ elsewhere. Extend $\hat{v}$ by reflection as above and again denote the result $\hat{v}$. In order to mollify $\hat{v}$, and thereby ensure that the result is contained in $\mathcal{A}_{i}$ for $i=1, \ldots, 3$, we first extend it by zero outside $[L-d, L] \times[0,4 a]$. Let $\psi$ be a standard mollifier function, let $\eta>0$, and set $\psi_{\eta}(\mathrm{x})=\frac{1}{\eta^{2}} \psi\left(\frac{\mathrm{x}}{\eta}\right)$ for $\mathrm{x} \in \mathbb{R}^{2}$. Define $\hat{v}_{\eta}=\psi_{\eta} * \hat{v}$ and note that since $0 \leq \psi \leq 1$, it follows that $\left|\hat{v}_{\eta, y}\right| \leq 1$ a.e. in $\mathbb{R}^{2}$. Moreover, $\mathcal{L}^{2}\left(B\left(\hat{v}_{\eta}\right)\right) \rightarrow \mathcal{L}^{2}(B(v))$ as $\eta \rightarrow 0$. One can now smoothly truncate $\hat{v}_{\eta}$ to ensure both that the result has compact support in $\Omega$ and that the set $B\left(\hat{v}_{\eta}\right)$ remains unchanged. The last statement of the proposition now follows by calculations similar to those given above and by letting $\eta \rightarrow 0$.

Remark 6. We remark that the proof of Proposition 4.3 above also shows that

$$
\frac{E_{1}^{\prime}(v)}{\mathcal{L}^{1}(\Pi(v))} \geq \frac{2 \lambda^{2} \epsilon^{\frac{3}{2}} C_{1}^{\frac{1}{4}} C_{2}^{\frac{3}{4}}}{d^{\frac{1}{2}}\left(1-\lambda^{-\frac{1}{2}}\right)}-4 \epsilon^{\frac{1}{2}}\left(\frac{C_{2}}{C_{1}}\right)^{\frac{1}{4}} \delta d^{\frac{1}{2}}\left(1-\lambda^{-\frac{1}{2}}\right) .
$$

By letting $d \rightarrow 0$, statement (a) holds. A version of statement (b) corresponding to setting $\sigma=0$ in (4.1) also holds. To see it, let $\lambda \rightarrow 1$ in the last inequality above while keeping $d$ (and hence a) fixed. 
4.3. Constructing $v$ such that $E_{3}^{\prime}(v) \rightarrow 0$ as $\mathcal{L}^{2}(B(v)) \rightarrow 0$. In this section it will be convenient to work with the functional $E_{3}$. The same results then hold for $E_{2}$ by using the relation (2.1). We seek a sequence $v^{(j)}$ of functions in $\mathcal{A}_{3}$ satisfying

(i) $\mathcal{L}^{2}\left(B\left(v^{(j)}\right)\right)>0$ for all $j$,

(ii) $\mathcal{L}^{2}\left(B\left(v^{(j)}\right)\right) \rightarrow 0$ as $j \rightarrow \infty$, and

(iii) $E_{3}\left(v^{(j)}\right)-E_{3}(0) \rightarrow 0+$ as $j \rightarrow \infty$.

One way to do this is to take advantage of the fact that if $f \in L^{2}(\Omega)$ and if $z$ is the Newtonian potential of $f$ (see, e.g., [12, Chapter 4]), then $\triangle z=f$ (where $\triangle$ denotes the Laplacian operator, as usual) and

$$
\int_{\Omega}\left|D^{2} z\right|^{2} \mathrm{dx}=\int_{\Omega}|f|^{2} \mathrm{dx} .
$$

(This is part of the Calderón-Zygmund theorem; see, e.g., [12, Theorem 9.9].) When $f$ is sufficiently smooth, for example, Lipschitz continuous, the representations

$$
z(\mathrm{x})=\int_{\Omega} \frac{1}{2 \pi} \ln (|\mathrm{x}-\mathrm{y}|) f(\mathrm{y}) \mathrm{dy}
$$

and

$$
\nabla z(\mathrm{x})=\int_{\Omega} \frac{1}{2 \pi} \frac{\mathrm{x}-\mathrm{y}}{|\mathrm{x}-\mathrm{y}|^{2}} f(\mathrm{y}) \mathrm{dy}
$$

hold. One now has control of both $\left\|D^{2} z\right\|_{2}$ and the pointwise behavior of $\nabla z$ via the function $f$. The next lemma details an appropriate choice of a sequence of functions $f^{(j)}$ whose corresponding potentials $z^{(j)}$ may be used to satisfy (i), (ii), and (iii) above. In the following we use the convention that $B_{r}(a)$ denotes the open ball of radius $r$ in $\mathbb{R}^{2}$ centered on $a$.

Lemma 4.4. Let the sequence of $L^{2}\left(B_{2}(0), \mathbb{R}\right)$ functions $f^{(j)}$ be defined by

$$
f^{(j)}(x, y)= \begin{cases}2^{j} A_{j} \frac{y}{R} & \text { if } 0<R \leq 2^{-j}, \\ A_{j} y R^{\alpha_{j}} & \text { if } 2^{-j} \leq R \leq 1, \\ A_{j} \frac{y}{R} & \text { if } 1 \leq R \leq 2,\end{cases}
$$

where

$$
\begin{aligned}
A_{j} & =\frac{k}{j}, \\
\alpha_{j} & =\frac{1}{j}-2, \\
R^{2} & =x^{2}+y^{2} .
\end{aligned}
$$

Let $\eta(R)$ be a smooth cut-off function with support in $B_{\frac{3}{2}}(0)$ such that $\eta(R)=1$ if $0 \leq R \leq 1$. Let $z^{(j)}$ be the Newtonian potential of $f^{(j)}$. Then the constant $k$ can be chosen so that

(a) $\left\|f^{(j)}\right\|_{2} \rightarrow 0$ as $j \rightarrow \infty$, and

(b) $z_{y}^{(j)}(0,0) \geq\left(L^{2}+1\right)^{\frac{1}{2}}$ for all sufficiently large $j$.

Here, $L$ is a positive constant. Let $\psi$ be a smooth cut-off function with support in $B_{\frac{3}{2}}(0)$ and which satisfies $\psi(R)=1$ if $0<R \leq 1$. Then the $C^{2}$ functions $\tilde{z}^{(j)}:=$ $\psi(R) z^{(j)}$ have compact support in $B_{2}(0)$ and they satisfy 
(c) $\tilde{z}_{y}^{(j)}(0,0) \geq\left(L^{2}+1\right)^{\frac{1}{2}}$ for all sufficiently large $j$, and

(d) $\int_{B_{2}(0)}\left|D^{2} \tilde{z}\right|^{2} \mathrm{dx} \rightarrow 0$ as $j \rightarrow \infty$.

Proof. (a) To see (a) we compute $\left\|f^{(j)}\right\|_{2}^{2}$ directly:

$$
\begin{aligned}
\int_{B_{2}(0)}\left(f^{(j)}\right)^{2} \mathrm{dx} & =\frac{\pi A_{j}^{2}}{2}+\pi A_{j}^{2} \int_{2^{-j}}^{1} R^{3+2 \alpha_{j}} d R+\frac{3 \pi A_{j}^{2}}{2} \\
& =2 \pi A_{j}^{2}+\frac{3 \pi j A_{j}^{2}}{16} \\
& =\frac{2 \pi k^{2}}{j^{2}}\left(1+\frac{3 j}{32}\right) .
\end{aligned}
$$

Now (a) follows easily (and independently of the choice of the constant $k$ ).

(b) To prove (b) first note that each $f^{(j)}$ is Lipschitz continuous, implying in particular that each $z^{(j)}$ is $C^{2}\left(B_{2}(0), \mathbb{R}\right)$ and that $(4.21)$ holds with $B_{2}(0)$ in place of $\Omega$. Thus

$$
\begin{aligned}
2 \pi z_{y}^{(j)}(0,0) & =\int_{B_{2}(0)}-\frac{y}{R} f^{(j)}(\mathrm{x}) \mathrm{dx} \\
& =-2^{j} \pi A_{j} \int_{0}^{2^{-j}} d R-\pi A_{j} \int_{2^{-j}}^{1} R^{\alpha_{j}+1} d R-\pi A_{j} \\
& =-2 \pi A_{j}-\frac{\pi k}{2} .
\end{aligned}
$$

Therefore

$$
z_{y}^{(j)}(0,0)=-\frac{k}{4}-A_{j}
$$

which on choosing $k=-6\left(L^{2}+1\right)^{\frac{1}{2}}$, say, and noting that $A_{j} \rightarrow 0$ as $j \rightarrow \infty$, implies $z_{y}^{(j)}(0,0) \geq\left(L^{2}+1\right)^{\frac{1}{2}}$ for all $j$. This is part (b) of the lemma.

(c) Part (c) follows easily from (b) and the definition of $\tilde{z}^{(j)}$ given above.

(d) By noting that

$$
\int_{B_{2}(0)}\left|D^{2} \tilde{z}^{(j)}\right| \mathrm{dx} \leq C \int_{B_{2}(0)}\left|z^{(j)}\right|^{2}+\left|\nabla z^{(j)}\right|^{2}+\left|D^{2} z^{(j)}\right|^{2} \mathrm{dx}
$$

for some constant $C$, it suffices to prove that $\int_{B_{2}(0)}\left|z^{(j)}\right|^{2} \mathrm{dx}$ and $\int_{B_{2}(0)}\left|\nabla z^{(j)}\right|^{2} \mathrm{dx}$ converge to zero as $j \rightarrow \infty$. The convergence to zero of the term $\int_{B_{2}(0)}\left|D^{2} z^{(j)}\right|^{2} \mathrm{dx}$ is guaranteed by (4.19) and part (a) above. By the representation (4.20), standard estimates, and Fubini's theorem,

$$
\int_{B_{2}(0)} z^{(j)^{2}} \mathrm{dx} \leq C \int_{B_{2}(0)}\left\{\int_{B_{2}(0)}(\ln (\mid \mathrm{x}-\mathrm{y}) \mid)^{2} \mathrm{dx}\right\} f^{(j)}(\mathrm{y})^{2} \mathrm{dy} .
$$

Therefore $\left\|z^{(j)}\right\|_{2}^{2} \rightarrow 0$ as $j \rightarrow \infty$. To check the convergence of $\left\|\nabla z^{(j)}\right\|_{2}$ to zero, write

$$
\int_{B_{2}(0)}\left|\nabla z^{(j)}\right|^{2} \mathrm{dx}=\int_{\partial B_{2}(0)} z^{(j)} \nabla z^{(j)} \cdot d \nu-\int_{B_{2}(0)} z^{j} \triangle z^{(j)} \mathrm{dx} .
$$

Next, note that both $z^{(j)}$ and $\nabla z^{(j)}$ converge uniformly to zero on $\partial B_{2}(0)$, which can be verified by using the fact that each $f^{(j)}$ has compact support in $B_{\frac{3}{2}}(0)$ together 
with the representations (4.20) and (4.21). The second term in (4.23) can be estimated by using Hölder's inequality and (4.19) in that order, giving

$$
\int_{B_{2}(0)}\left|z^{(j)} \triangle z^{(j)}\right| \mathrm{dx} \leq C|| f^{(j)} \|_{2}^{2}
$$

for some generic constant $C$. Therefore $\left\|\nabla z^{(j)}\right\|_{2} \rightarrow 0$, which concludes the proof.

The next result formalizes the statement made at the start of this subsection. The proof can easily be adapted to show that sets $B(v)$ of positive measure can be introduced into $\Omega$ in such a way that $E_{2}(v)-E_{2}(0)$ can be made arbitrarily small.

THeOREM 4.5. Let $E_{3}$ and $\mathcal{A}_{3}$ be as per (1.3) and (1.7), respectively. Then there exist sequences $\left\{v^{(j)}\right\} \subset \mathcal{A}_{3}$ such that

(i) $\mathcal{L}^{2}\left(B\left(v^{(j)}\right)\right)>0$ for all sufficiently large $j$,

(ii) $\mathcal{L}^{2}\left(B\left(v^{(j)}\right)\right) \rightarrow 0$ as $j \rightarrow \infty$, and

(iii) $E_{3}\left(v^{(j)}\right)-E_{3}(0) \rightarrow 0$ as $j \rightarrow \infty$.

In other words, sets $B(v)$ of positive measure can be introduced at arbitrarily small energies, as measured by $E_{3}$.

Proof. Let $P$ be the point $\left(\frac{L}{2}, \frac{1}{2}\right)$ in $\Omega$ and define the planar affine map $T$ by

$$
T(x)=\frac{2(x-P)}{\left(L^{2}+1\right)^{\frac{1}{2}}} .
$$

Then $T(\Omega) \subset B_{2}(0), \operatorname{lip}(T)=\frac{2}{\left(L^{2}+1\right)^{\frac{1}{2}}}$, and we can define

$$
v^{(j)}(x)=\tilde{z}^{(j)}(T(x))
$$

for $x \in \Omega$, where $\tilde{z}^{(j)}$ is as per Lemma 4.4. To check that (i) holds is now straightforward. Indeed, since

$$
v_{y}^{(j)}(x)=\operatorname{lip}(T) \tilde{z}_{y}^{(j)}(T(x))
$$

for all $x$, it follows from part (c) of Lemma 4.4 that

$$
v_{y}^{(j)}(p)=\operatorname{lip}(T) \tilde{z}^{(j)}(0) \geq 2
$$

Since $v^{(j)}$ is $C^{2}$, it follows that $\mathcal{L}^{2}\left(\left\{\left|v_{y}^{(j)}\right| \geq 1\right\}\right)>0$, which is statement (i) above. To see statements (ii) and (iii) note that

$$
\begin{aligned}
E_{3}\left(v^{(j)}\right) & =\int_{\Omega} \epsilon^{2}\left|D^{2} v^{(j)}\right|^{2}+v_{x}^{(j)^{2}} \mathrm{dx}+\delta \mathcal{L}^{2}\left(B\left(v^{(j)}\right)\right) \\
& \leq \int_{B_{2}(0)} \epsilon^{2}(\operatorname{lip}(T))^{2}\left|D^{2} \tilde{z}^{(j)}\right|^{2}+\left(\tilde{z}_{x}^{(j)}\right)^{2} \mathrm{dy}+C \int_{\Omega}\left(\tilde{z}_{y}^{(j)}\right)^{2} \mathrm{dy} \\
& \leq C \int_{B_{2}(0)}\left|D^{2} \tilde{z}^{(j)}\right|^{2}+\left|\nabla \tilde{z}^{(j)}\right|^{2} \mathrm{dy},
\end{aligned}
$$

where we have applied Chebyshev's inequality in the second line. The right-hand side can now be made arbitrarily small by appealing to part (d) of Lemma 4.4, proving parts (ii) and (iii) of the theorem.

Remark 7. It would be of interest to compute $|\Pi(v)|$ for the $v$ constructed in section 4.3. One could then compare the quantity $\frac{E_{3}^{\prime}(v)}{|\Pi(v)|^{\sigma+1}}$ with the lower bound predicted in part (b) of Theorem 4.1. 
Appendix. This section is included for completeness only. We show that the global minimizer of $E_{i}$ in $\mathcal{A}_{i}$ exists. The case $i=2$ is considered here; the others follow by analogy.

Recall that

$$
E_{2}(v)=\int_{\Omega}\left|\nabla\left(v_{y}\right)\right|^{2}+v_{x}^{2} \mathrm{dx}+\delta \mathcal{L}^{2}(A(v))
$$

where

$$
A(v)=\left\{(x, y) \in \mathbb{R}^{2}:\left|v_{y}(x, y)\right|<1\right\} .
$$

Now $E_{2}$ is bounded below by zero, so its infimum in $\mathcal{A}_{2}$ exists. Let $\left\{v^{(j)}\right\} \subset \mathcal{A}_{2}$ be a minimizing sequence. Then, since $\nabla\left(v_{y}^{(j)}\right)$ is bounded in $L^{2}$, it follows that there is $w$ in $L^{2}$ such that for a subsequence (and after relabeling) $v_{y}^{(j)} \rightarrow w$ in $W^{1,2}$. By the Rellich-Kondrachov compactness theorem, we can suppose that the sequence $v_{y}^{(j)}$ converges strongly, and is in particular bounded, in $L^{2}$. Since $E_{2}\left(v^{(j)}\right)$ is bounded, it follows that $v_{x}^{(j)}$ is also bounded in $L^{2}$. Therefore $\left|\nabla v^{(j)}\right|$ is bounded in $L^{2}$, and hence there is some function $v$ in $W^{1,2}(\Omega, \mathbb{R})$ such that

$$
v^{(j)} \rightarrow v \text { in } W^{1,2} .
$$

It follows that $w=v_{y}$. The trace theorems for Sobolev functions now imply that $v \in \mathcal{A}_{2}$. (See [10, section 4.3, Theorem 1], or (A.5) in the appendix of [14], for example.) The sequential lower semicontinuity of

$$
v \mapsto \int_{\Omega} v_{x}^{2} \mathrm{dx}
$$

and of

$$
v_{y} \mapsto \int_{\Omega}\left|\nabla\left(v_{y}\right)\right|^{2} \mathrm{dx}
$$

with respect to weak convergence in $W^{1,2}$ follows from standard arguments using the convexity of the integrands. We may assume that $v_{y}^{(j)} \rightarrow v_{y}$ a.e. in $\Omega$. Let $f^{(j)}=\chi_{A\left(v^{(j)}\right)}$ and $f=\chi_{A(v)}$. Since the inequality defining membership of the set $A(v)$ is strict, it follows that $\liminf _{j \rightarrow \infty} f^{(j)} \geq f$ a.e. Hence, by Fatou's lemma,

$$
\liminf _{j \rightarrow \infty} \mathcal{L}^{2}\left(A\left(v^{(j)}\right)\right) \geq \mathcal{L}^{2}(A(v)) .
$$

In summary, $\liminf _{j \rightarrow \infty} E_{2}\left(v^{(j)}\right) \geq E_{2}(v)$, which concludes the proof of the existence of the global minimizer $v$ of $E_{2}$ in $\mathcal{A}_{2}$.

Acknowledgments. I would like to thank Prof. John Ball for showing me a version of [2, Theorem 2.2], and Prof. Stefan Müller for sparking my interest in this problem.

\section{REFERENCES}

[1] J. M. BALL, Plenary talk at conference, Multi-scale Problems: Modelling, Analysis and Applications, University of Bath, 2005. 
[2] J. M. Ball And E. C. M. Crooks, Local minimizers in a phase-transition model with interfacial energy, Calc. Var. Partial Differential Equations, 40 (2011), pp. 501-538.

[3] J. M. Ball And R. D. James, Fine phase mixtures as minimizers of energy, Arch. Rational Mech. Anal., 100 (1987), pp. 13-52.

[4] J. M. Ball And R. D. James, Proposed experimental tests of a theory of fine microstructure and the two well problem, Philos. Trans. R. Soc. Lond. Ser. A Math. Phys. Eng. Sci., 338 (1992), pp. 389-450.

[5] J. M. Ball, R. J. Knops, and J. E. Marsden, Two examples in nonlinear elasticity, in Journées d'Analyse Non Linéaire (Proc. Conf., Besancon, 1977), Lecture Notes in Math. 665, Springer, Berlin, 1978, pp. 41-48.

[6] J. M. BAll AND J. E. MARSDEn, Quasiconvexity at the boundary, positivity of the second variation and elastic stability, Arch. Rational Mech. Anal., 86 (1984), pp. 251-277.

[7] J. M. Ball And C. Mora-Corral, A variational model allowing both smooth and sharp phase boundaries in solids, Commun. Pure Appl. Anal., 8 (2009), pp. 55-81.

[8] S. Conti, I. Fonseca, And G. LeOni, A $\Gamma$-convergence result for the two-gradient theory of phase transitions, Comm. Pure Appl. Math., 55 (2002), pp. 857-936.

[9] S. Conti AND B. SChweIzer, Rigidity and gamma convergence for solid-solid phase transitions with $S O(2)$ invariance, Comm. Pure Appl. Math., 59 (2006), pp. 830-868.

[10] L. C. Evans And R. F. Gariepy, Measure theory and fine properties of functions, Stud. Adv. Math., CRC Press, Boca Raton, FL, 1992.

[11] I. Fonseca And C. Mantegazza, Second order singular perturbation models for phase transitions, SIAM J. Math. Anal., 31 (2000), pp. 1121-1143.

[12] D. Gilbarg and N. S. Trudinger, Elliptic Partial Differential Equations of Second Order, Classics in Mathematics, Springer-Verlag, Berlin, 2001. Reprint of the 1998 edition.

[13] R. V. KoHn, Energy-driven pattern formation, in International Congress of Mathematicians. Vol. I, Eur. Math. Soc., Zürich, 2007, pp. 359-383.

[14] R. V. Kohn ANd S. Müller, Surface energy and microstructure in coherent phase transitions, Comm. Pure Appl. Math., 47 (1994), pp. 405-435.

[15] S. MüLleR, Variational models for microstructure and phase transitions, in Calculus of Variations and Geometric Evolution Problems (Cetraro, 1996), Lecture Notes in Math. 1713, Springer, Berlin, 1999, pp. 85-210.

[16] A. TAHERI, Strong versus weak local minimizers for the perturbed Dirichlet functional, Calc. Var. Partial Differential Equations, 15 (2002), pp. 215-235.

[17] W. P. Ziemer, Weakly Differentiable Functions. Sobolev Spaces and Functions of Bounded Variation, Grad. Texts in Math. 120, Springer-Verlag, New York, 1989. 